\title{
A CHARACTERIZATION OF RIGHT 4-NAKAYAMA ARTIN ALGEBRAS
}

\author{
ALIREZA NASR-ISFAHANI AND MOHSEN SHEKARI
}

\begin{abstract}
We characterize right 4-Nakayama artin algebras which appear naturally in the study of representation-finite artin algebras. For a right 4-Nakayama artin algebra $\Lambda$, we classify all finitely generated indecomposable right $\Lambda$-modules and then we compute all almost split sequences over $\Lambda$. We also give a characterization of right 4-Nakayama finite dimensional $K$-algebras in terms of their quivers with relations.
\end{abstract}

\section{INTRODUCTION}

Let $R$ be a commutative artinian ring. An artin algebra is an $R$-algebra $\Lambda$ that is a finitely generated $R$-module. Let $\Lambda$ be an artin algebra. A right $\Lambda$-module $M$ is called uniserial (1-factor serial) if the lattice of its submodules is totally ordered under inclusion. An artin algebra $\Lambda$ is called Nakayama algebra if the indecomposable projective right $\Lambda$-modules as well as the indecomposable projective left $\Lambda$-modules are uniserial. This then implies that all the finitely generated indecomposable right $\Lambda$-modules are uniserial. Nakayama algebras are representation-finite algebras whose module categories completely understood (see [3], [7] and [11]). A non-uniserial right $\Lambda$-module $M$ of length $l$ is called $n$-factor serial $(l \geq n>1)$, if $\frac{M}{\operatorname{rad}^{l-n}(M)}$ is uniserial and $\frac{M}{\operatorname{rad}^{l-n+1}(M)}$ is not uniserial ([6, Definition 2.1]). An artin algebra $\Lambda$ is called right $n$-Nakayama if every indecomposable right $\Lambda$-module is $i$-factor serial for some $1 \leq i \leq n$ and there exists at least one indecomposable $n$-factor serial right $\Lambda$-module [6, Definition 2.2]. The authors in [6] proved that an artin algebra $\Lambda$ is representation-finite if and only if $\Lambda$ is right $n$-Nakayama for some positive integer $n$ [6, Theorem 2.18]. Indecomposable modules and almost split sequences over right 2-Nakayama and right 3-Nakayama artin algebras are studied in [6] and [5]. In this paper we will study the module categories of right 4-Nakayama algebras. We first show that an artin algebra $\Lambda$ which is neither Nakayama nor right 2-Nakayama nor right 3 -Nakayama is right 4-Nakayama if and only if every indecomposable right $\Lambda$-module of length greater than 5 is uniserial and every indecomposable right $\Lambda$-module of length 5 is local. Then we classify all indecomposable modules and almost split sequences over a right 4-Nakayama artin algebra. We also show that finite dimensional right 4-Nakayama algebras are special biserial and characterize quivers and relations of finite dimensional right 4-Nakayama algebras. The paper is organized as follows. In Section 2 we study 4 -factor serial right $\Lambda$-modules and then we give a characterization of right 4-Nakayama artin algebras.

2000 Mathematics Subject Classification. 16G20, 16G60, 16G70, 16D70, 16D90.

Key words and phrases. Right 4-Nakayama algebras, Almost split sequences, Indecomposable modules, Special biserial algebras. 
In Section 3 we classify all indecomposable modules over right 4-Nakayama artin algebras, up to isomorphisms.

In section 4 we compute all almost split sequences over right 4-Nakayama artin algebras.

Finally in the last section we describe the structure of quivers and their relations of finite dimensional right 4-Nakayama algebras.

1.1. notation. Throughout this paper all modules are finitely generated right $\Lambda$-modules and all fields are algebraically closed fields. For a $\Lambda$-module $M$, we denote by $\operatorname{soc}(M)$, top $(M), \operatorname{rad}(M), l(M)$ and $l l(M)$ its socle, top, radical, length and Loewy length of $M$, respectively. Let $Q=\left(Q_{0}, Q_{1}, s, t\right)$ be a quiver and $\alpha: i \rightarrow j$ be an arrow in $Q$. One introduces a formal inverse $\alpha^{-1}$ with $s\left(\alpha^{-1}\right)=j$ and $t\left(\alpha^{-1}\right)=i$. An edge in $Q$ is an arrow or the inverse of an arrow. To each vertex $i$ in $Q$, one associates a trivial path, also called trivial walk, $\varepsilon_{i}$ with $s\left(\varepsilon_{i}\right)=t\left(\varepsilon_{i}\right)=i$. A non-trivial walk $w$ in $Q$ is a sequence $w=c_{1} c_{2} \cdots c_{n}$, where the $c_{i}$ is an edge such that $t\left(c_{i}\right)=s\left(c_{i+1}\right)$ for all $i$, whose inverse $w^{-1}$ is defined to be the sequence $w^{-1}=c_{n}^{-1} c_{n-1}^{-1} \cdots c_{1}^{-1}$. A walk $w$ is called reduced if $c_{i+1} \neq c_{i}^{-1}$ for each $i$. For $i \in Q_{0}$, we denote by $i^{+}$and $i^{-}$the set of arrows starting in $i$ and the set of arrows ending in $i$, respectively.

\section{RIGHT 4-NAKAYAMA ARTIN ALGEBRAS}

In this section we first describe right 4-factor serial modules and then we give a characterization of right 4-Nakayama artin algebras.

Definition 2.1. [6, Definitions 2.1 and 2.2] Let $\Lambda$ be an artin algebra and $M$ be a right $\Lambda$-module of length $l$.

(1) $M$ is called 1-factor serial (uniserial) if $M$ has a unique composition series.

(2) Let $l \geq n>1$. $M$ is called $n$-factor serial if $\frac{M}{\operatorname{rad}^{l-n}(M)}$ is uniserial and $\frac{M}{\operatorname{rad}^{l-n+1}(M)}$ is not uniserial.

(3) $\Lambda$ is called right $n$-Nakayama if every indecomposable right $\Lambda$-module is $i$-factor serial for some $1 \leq i \leq n$ and there exists at least one indecomposable $n$-factor serial right $\Lambda$-module.

Lemma 2.2. Let $\Lambda$ be an artin algebra and $M$ be a right $\Lambda$-module of length $r$ and Loewy length $t$. The following conditions are equivalent.

(a) $M$ is a 4-factor serial right $\Lambda$-module.

(b) One of the following conditions hold.

(i) For every $0 \leq i \leq r-5$, $\operatorname{rad}^{i}(M)$ is local, $\operatorname{rad}^{r-4}(M)$ is not local and length of $M$ is either $t+3$ or $t+2$ or $t+1$. Moreover in this case if $r=t+3$, then $\operatorname{rad}^{r-4}(M)=\operatorname{soc}(M)=S_{1} \oplus S_{2} \oplus S_{3} \oplus S_{4}$, where $S_{i}$ is a simple submodule of $M$ for each $1 \leq i \leq 4$.

(ii) $M$ is not local, $r=4$ and the Loewy length of $M$ is either 2 or 3 . Moreover in this case if $\operatorname{soc}(M)$ is simple, then $l l(M)=3$, otherwise $l l(M)=2$.

Proof. $(a) \Longrightarrow(b)$. Assume that $M$ is a local right $\Lambda$-module. Then by [6, Theorem 2.6], for every $0 \leq i \leq r-5, \operatorname{rad}^{i}(M)$ is local and $\operatorname{rad}^{r-4}(M)$ is not local. On the other hand by [6. Lemma 2.21], $t+1 \leq r \leq t+3$. If $r=t+3$, then by [6, Remark 2.7], 
$\operatorname{soc}(M) \subseteq \operatorname{rad}^{r-4}(M)$ and $t=r-3$. Therefore $\operatorname{soc}(M)=\operatorname{rad}^{r-4}(M)=S_{1} \oplus S_{2} \oplus S_{3} \oplus S_{4}$. This finishes the proof of $(i)$. Assume that $M$ is not local. So by [6, Corollary 2.8], $r=4$ and the result follows.

$(b) \Longrightarrow(a)$. If $M$ is not local and $r=4$, then by [6, Corollary 2.8], $M$ is a 4 -factor serial right $\Lambda$-module. If $M$ satisfies the condition $(i)$, then $\frac{M}{\operatorname{rad}^{r-4}(M)}$ is uniserial and $\frac{M}{\mathrm{rad}^{r-3}(M)}$ is not uniserial. Therefore $M$ is a 4 -factor serial right $\Lambda$-module

An Artin algebra $\Lambda$ is of right $n$-th local type if for every indecomposable right $\Lambda$-module $M, \operatorname{top}^{n}(M)=\frac{M}{\operatorname{rad}^{n}(M)}$ is indecomposable [2].

Lemma 2.3. Let $\Lambda$ be a right 4-Nakayama artin algebra. Then $\Lambda$ is of 3-ed local type.

Proof. Let $M$ be an indecomposable right $\Lambda$-module. If $M$ is local, then $\frac{M}{\operatorname{rad}^{3}(M)}$ is indecomposable. If $M$ is not local, then by [6, Lemma 5.2], $M$ is either 3 -factor serial or 4-factor serial. By [5, Lemma 2.1] and Lemma 2.2, $l l(M) \leq 3$, so $\frac{M}{\operatorname{rad}^{3}(M)} \cong M$ is indecomposable and the result follows.

Let $\Lambda$ be an artin algebra. The valued quiver of $\Lambda$ is a quiver with $n$ vertices, where $n$ is the number of the isomorphism classes of simple right $\Lambda$-modules and with at most one arrow from a vertex $i$ to the vertex $j$ and with an ordered pair of positive integers associated with each arrow. This is done by writing an arrow from $i$ to $j$ if $\operatorname{Ext}_{\Lambda}^{1}\left(S_{i}, S_{j}\right) \neq 0$, where $S_{i}$ and $S_{j}$ are simple $\Lambda$-modules corresponding to the vertices $i$ and $j$ and assigning

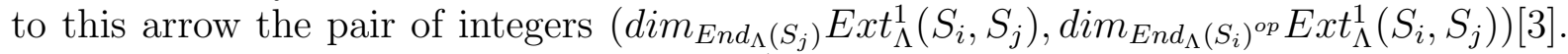
Note that by [3. Proposition III.1.15], $\operatorname{Ext}_{\Lambda}^{1}\left(S_{i}, S_{j}\right) \neq 0$ if and only if $S_{j}$ is a direct summand of $\frac{\operatorname{rad}\left(P_{i}\right)}{\operatorname{rad}^{2}\left(P_{i}\right)}$, where $P_{i}$ is a projective cover of $S_{i}$.

Let $\Lambda$ be an artin algebra and $M$ be a right $\Lambda$-module. $M$ has a waist if there is a nontrivial proper submodule $N$ of $M$ such that every submodule of $M$ contains $N$ or is contained in $N$. In this case, $N$ is called a waist in $M$.

Proposition 2.4. Let $\Lambda$ be a right 4-Nakayama artin algebra and $M$ be an indecomposable local right $\Lambda$-module. Then the following statements hold.

(a) If $M$ is 4-factor serial, then $l(M)=5$.

(b) If $M$ is 3-factor serial, then $l(M)=4$.

(c) If $M$ is 2-factor serial, then $l(M)=3$.

Proof. (a). By [5, Lemma 2.3], $M$ is a projective right $\Lambda$-module and by Lemma 2.3, $\Lambda$ is of 3-ed local type. If $\operatorname{rad}^{3}(M) \neq 0$, then by [1, Theorem 2.5], $\operatorname{rad}^{3}(M)$ is uniserial and waist of $M$. Also by [6, Theorem 2.6], $\operatorname{rad}(M)$ is 4 -factor serial. Assume that $\operatorname{rad}(M)$ is local. Then by [6, Theorem 2.6], $\operatorname{rad}^{2}(M)$ is 4 -factor serial. If $\operatorname{rad}^{2}(M)$ is local, then by [6, Theorem 2.6], $\operatorname{rad}^{3}(M)$ is 4-factor serial which gives a contradiction. Therefore $\operatorname{rad}^{2}(M)$ is non-local and by [6, Corollary 2.8] $l\left(\operatorname{rad}^{2}(M)\right)=4$. This implies that $l(M)=6$. Let top $(M)=S_{1}$, top $(\operatorname{rad}(M))=S_{2}$ and $S_{3}, S_{4}$ be direct summands of top $\left(\operatorname{rad}^{2}(M)\right)$. Since $M$ is projective, by [3, Proposition III.1.15], there exists one arrow from the vertex 1 to the vertex 2 in the valued quiver of $\Lambda$. Since $\operatorname{rad}(M)$ is local, $\operatorname{rad}(M)$ is either projective or quotient of an indecomposable projective right $\Lambda$-module $P_{2}$. Assume that $\operatorname{rad}(M)$ is projective. Since $S_{3}$ and $S_{4}$ are direct summands of $t o p\left(\operatorname{rad}^{2}(M)\right)$, by [3, Proposition 
III.1.15], there are one arrow from the vertex 2 to the vertex 3 and one arrow from the vertex 2 to the vertex 4 in the valued quiver of $\Lambda$. Then the valued quiver of $\Lambda$ has a subquiver of the form

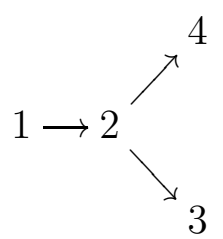

which implies that there exists a non-local indecomposable right $\Lambda$-module $N$ of length 5. Therefore by [6, Corollary 2.8], $N$ is a 5 -factor serial right $\Lambda$-module which is a contradiction. Now assume that $\operatorname{rad}(M)$ is not projective. Then $\operatorname{rad}(M) \cong \frac{P_{2}}{K}$, where $P_{2}$ is the indecomposable projective right $\Lambda$-module and $K$ is a submodule of $P_{2}$ and so $\operatorname{rad}^{2}(M) \cong \frac{\operatorname{rad}\left(P_{2}\right)}{K}$. Then $S_{3}$ and $S_{4}$ are direct summands of $\operatorname{rad}\left(P_{2}\right)$ and by $[3$, Proposition III.1.15], there are one arrow from the vertex 2 to the vertex 3 and one arrow from the vertex 2 to the vertex 4 in the valued quiver of $\Lambda$. Then the valued quiver of $\Lambda$ has a subquiver of the form

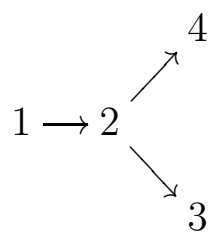

which implies that there exists a non-local indecomposable right $\Lambda$-module $N$ of length 5 . Then by [6, Corollary 2.8], $N$ is a 5 -factor serial right $\Lambda$-module which is a contradiction. Therefore $\operatorname{rad}(M)$ is non-local and by [6, Corollary 2.8], $\operatorname{rad}(M)$ is of the length 4 . It implies that $l(M)=5$.

In the parts $(b)$ and $(c), M$ is local. Then $M$ is either projective or quotient of an indecomposable projective. Then by the similar argument as in the proof of the part $(a)$, we can prove parts $(b)$ and $(c)$.

Now we give a characterization of right 4-Nakayama artin algebras.

Theorem 2.5. Let $\Lambda$ be an artin algebra which is neither Nakayama, nor right 2-Nakayama, nor right 3-Nakayama. Then $\Lambda$ is right 4-Nakayama if and only if every indecomposable right $\Lambda$-module $M$ of length greater than 5 is uniserial and every indecomposable right $\Lambda$-module $M$ of length 5 is local.

Proof. Let $\Lambda$ be a right 4-Nakayama algebra. It follows from Proposition 2.4 that, every indecomposable right $\Lambda$-module $M$ of length greater than 5 is uniserial. If there exists an indecomposable non-local right $\Lambda$-module $M$ of length 5 , then by [6, Corollary 2.8], $M$ is 5 -factor serial which gives a contradiction. Conversely, assume that any indecomposable right $\Lambda$-module of length greater than 5 is uniserial and every indecomposable right $\Lambda$-module of length 5 is local, so by [6, Corollary 2.8] and [6, Lemma 2.21], every indecomposable right $\Lambda$-module is $t$-factor serial for some $t \leq 4$. Since $\Lambda$ is neither Nakayama, nor right 2-Nakayama, nor right 3 -Nakayama, there exists an indecomposable $t$-factor serial right $\Lambda$-module $M$ for some $t \geq 4$. Therefore $\Lambda$ is right 4 -Nakayama. 


\section{INDECOMPOSABLE MODULES OVER RIGHT 4-NAKAYAMA ARTIN ALGEBRAS}

In this section we give a classification of finitely generated indecomposable modules over right 4-Nakayama artin algebras.

Proposition 3.1. Let $\Lambda$ be a right 4-Nakayama artin algebra and $M$ be an indecomposable right $\Lambda$-module. Then $l(\operatorname{soc}(M)) \leq 2$.

Proof. If $M$ is uniserial, then $l(\operatorname{soc}(M))=1$ and if $M$ is 2-factor serial, then by [6, Lemma 5.1], $l(\operatorname{soc}(M))=2$. Assume that $M$ is 3 -factor serial. If $M$ is non-local, then $l(M)=3$ and by [5, Lemma 2.1], $l(\operatorname{soc}(M))=1$ and if $M$ is local, then by Proposition 2.4, $l(M)=4$ and $l(\operatorname{soc}(M)) \leq 3$. Assume that on the contrary that $\operatorname{rad}(M)=\operatorname{soc}(M)=S_{1} \oplus S_{2} \oplus S_{3}$ and $\operatorname{top}(M)=S_{4}$, where $S_{i}$ is a simple $\Lambda$-module for each $1 \leq i \leq 4$. Then by the similar argument as in the proof of the Proposition 2.4, the valued quiver of $\Lambda$ has a subquiver of the form

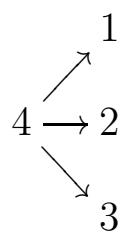

Then there exists a non-local indecomposable right $\Lambda$-module of length 5 that by $[\underline{6}$, Corollary 2.8] is 5-factor serial which gives a contradiction. Now assume that $M$ is 4 factor serial. If $M$ is non-local, then by Lemma 2.2, $l(M)=4$ and since $l(\operatorname{top}(M)) \geq 2$, $l(\operatorname{soc}(M)) \leq 2$. If $M$ is local, then by [5, Lemma 2.2], $M$ is projective and by Proposition 2.4. $l(M)=5$. Assume on the contrary that $l(\operatorname{soc}(M))=4$. Then $\operatorname{rad}(M)=\operatorname{soc}(M)=$ $S_{1} \oplus S_{2} \oplus S_{3} \oplus S_{4}$, where $S_{i}$ is a simple $\Lambda$-module for each $1 \leq i \leq 4$. Therefore we have almost split sequences

$$
\begin{gathered}
0 \longrightarrow S_{1} \longrightarrow M \longrightarrow \tau^{-1}\left(S_{1}\right) \longrightarrow 0 \\
0 \longrightarrow S_{2} \longrightarrow M \longrightarrow \tau^{-1}\left(S_{2}\right) \longrightarrow 0 \\
0 \longrightarrow S_{3} \longrightarrow M \longrightarrow \tau^{-1}\left(S_{3}\right) \longrightarrow 0 \\
0 \longrightarrow S_{4} \longrightarrow M \longrightarrow \tau^{-1}\left(S_{4}\right) \longrightarrow 0 \\
0 \rightarrow M \rightarrow \tau^{-1}\left(S_{1}\right) \oplus \tau^{-1}\left(S_{2}\right) \oplus \tau^{-1}\left(S_{3}\right) \oplus \tau^{-1}\left(S_{4}\right) \rightarrow \tau^{-1}(M) \rightarrow 0
\end{gathered}
$$

Then $\tau^{-1}(M)$ is an indecomposable right $\Lambda$-module of length 11 and by Proposition 2.4 , $\tau^{-1}(M)$ is uniserial. Also for each $1 \leq i \leq 4$, irreducible morphisms $\tau^{-1}\left(S_{i}\right) \longrightarrow \tau^{-1}(M)$ are monomorphisms. For each $1 \leq i \leq 4, \tau^{-1}\left(S_{i}\right) \cong \frac{M}{S_{i}}$ and by [6, Theorem 2.13], there exists $1 \leq i \leq 4$ such that $\tau^{-1}\left(S_{i}\right) \cong \frac{M}{S_{i}}$ is not uniserial which gives a contradiction to the Corollary 2.17 of [6]. Now assume that $l(\operatorname{soc}(M))=3$ and $\operatorname{soc}(M)=S_{1} \oplus S_{2} \oplus S_{3}$ where $S_{i}$ is a simple $\Lambda$-module for each $1 \leq i \leq 3$. If $\operatorname{rad}(M)=N \oplus S_{2} \oplus S_{3}$, where $N$ is uniserial of length 2 and $\operatorname{top}(N)=S_{4}, \operatorname{soc}(N)=S_{1}$ and $\operatorname{top}(M)=S_{5}$, then by the similar 
argument as in the proof of the Proposition 2.4, the valued quiver of $\Lambda$ has a subquiver of the form

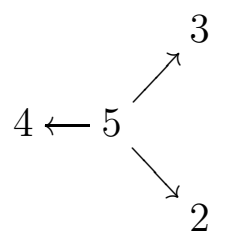

Therefore there exists a non-local indecomposable right $\Lambda$-module $L$ of length 5 . By [6, Corollary 2.8], $L$ is 5-factor serial which gives a contradiction. If $\operatorname{rad}(M)=N \oplus S_{3}$ where $N$ is a 2-factor serial right $\Lambda$-module of length $3, \operatorname{top}(N)=S_{4}$ and $\operatorname{rad}(N)=\operatorname{soc}(N)=$ $S_{1} \oplus S_{2}$. The similar argument as in the proof of Proposition 2.4 shows that the valued quiver of $\Lambda$ has a subquiver of the form

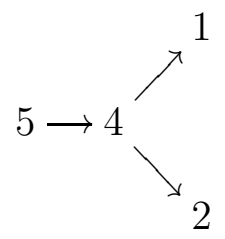

which gives a contradiction. Therefore $l(\operatorname{soc}(M)) \leq 2$ and the result follows.

Proposition 3.2. Let $\Lambda$ be a right 4-Nakayama artin algebra and $M$ be an indecomposable 4-factor serial right $\Lambda$-module. Then the following statements hold.

(a) If $M$ is local and $\operatorname{rad}(M)$ is indecomposable, then soc $(M)$ is simple.

(b) If $M$ is non-local, then $l(\operatorname{top}(M))=2$.

Proof. (a). By [5, Lemma 2.3], $M$ is projective and by the Proposition 2.4, $l(M)=5$. By the Proposition [3.1, $l(\operatorname{soc}(M)) \leq 2$ and by [6, Theorem 2.6, Corollary 2.8], $\operatorname{rad}(M)$ is nonlocal 4-factor serial of length 4. Assume that $\operatorname{soc}(M)=S_{1} \oplus S_{2}, \operatorname{top}(\operatorname{rad}(M))=S_{3} \oplus S_{4}$ and $\operatorname{top}(M)=S_{5}$, where $S_{i}$ is a simple $\Lambda$-module for each $1 \leq i \leq 5$. Since $M$ is projective, by [3. Proposition III.1.15] there are one arrow from the vertex 5 to the vertex 3 and one arrow from the vertex 5 to the vertex 4 in the valued quiver of $\Lambda$. Since $\operatorname{top}(\operatorname{rad}(M))=S_{3} \oplus S_{4}$, $\frac{P_{3} \oplus P_{4}}{L} \cong \operatorname{rad}(M)$ where $P_{i}$ is indecomposable projective that $\operatorname{top}\left(P_{i}\right)=S_{i}$ and $L$ is a submodule of $P_{3} \oplus P_{4}$. Then $S_{1} \oplus S_{2}=\operatorname{soc}(M)=\frac{\operatorname{rad}(M)}{\operatorname{rad}^{2}(M)} \subseteq \frac{\operatorname{rad}\left(P_{3} \oplus P_{4}\right)}{\operatorname{rad}^{2}\left(P_{3} \oplus P_{4}\right)}$. Since $\operatorname{rad}(M)$ is indecomposable, $S_{1} \oplus S_{2}$ must be a direct summand of either $\operatorname{top}\left(\operatorname{rad}\left(P_{3}\right)\right)$ or top $\left(\operatorname{rad}\left(P_{4}\right)\right)$. We can assume that $S_{1} \oplus S_{2}$ is a direct summand of $\operatorname{top}\left(\operatorname{rad}\left(P_{3}\right)\right)$. By [3. Proposition III.1.15], there are one arrow from the vertex 3 to the vertex 2 and one arrow from the vertex 3 to the vertex 1 in the valued quiver of $\Lambda$. Then the valued quiver of $\Lambda$ has a subquiver of the form

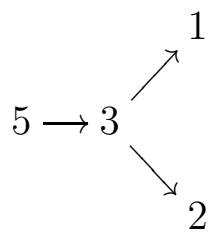

which implies that there is a non-local indecomposable right $\Lambda$-module $N$ of length 5 . By [6. Corollary 2.8], $N$ is 5-factor serial which gives a contradiction. Therefore $\operatorname{soc}(M)$ is simple. 
(b). By Lemma 2.2, $l(M)=4$. Assume on the contrary that $\operatorname{top}(M)=S_{1} \oplus S_{2} \oplus S_{3}$ and $\operatorname{soc}(M)=S_{4}$, where $S_{i}$ is a simple $\Lambda$-module for each $1 \leq i \leq 4$. $M$ has a projective cover of the form $f=\left(f_{1}, f_{2}, f_{3}\right): P_{1} \oplus P_{2} \oplus P_{3} \rightarrow M$. Then $\frac{P_{1} \oplus P_{2} \oplus P_{3}}{L} \cong M$, where $L=\operatorname{Ker}(f)$ and $S_{4}=\operatorname{rad}(M) \cong \frac{\operatorname{rad}\left(P_{1}\right) \oplus \operatorname{rad}\left(P_{2}\right) \oplus \operatorname{rad}\left(P_{3}\right)}{L}$. We claim that for each $1 \leq i \leq 3$, $S_{4}$ is a direct summand of $\operatorname{rad}\left(P_{i}\right)$. Assume that $S_{4}$ is not a direct summand of $\operatorname{rad}\left(P_{1}\right)$. Since $S_{4}=\operatorname{rad}(M), \operatorname{rad}\left(P_{1}\right) \subset L$. We consider the $\Lambda$-homomorphism $f_{1}: P_{1} \rightarrow M$. Then $\frac{P_{1}}{\operatorname{rad}\left(P_{1}\right)} \cong I m f_{1} \leq M$. Since $\frac{P_{1}}{\operatorname{rad}\left(P_{1}\right)} \cong I m f_{1}$ is simple, $\operatorname{Im} f_{1}$ is direct summand of $\operatorname{soc}(M)=S_{4}$ and $I m f_{1}=S_{4}$ which gives a contradiction. The similar argument shows that $S_{4}$ is a direct summand of $\operatorname{rad}\left(P_{2}\right)$ and $\operatorname{rad}\left(P_{3}\right)$. So by [3, Proposition III.1.15], the valued quiver of $\Lambda$ has a subquiver of the form

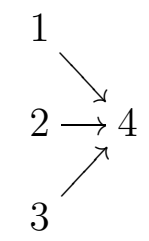

which gives a contradiction.

The following theorem gives a classification of submodules of indecomposable modules over right 4-Nakayama artin algebras.

Theorem 3.3. Let $\Lambda$ be a right 4-Nakayama artin algebra and $M$ be an indecomposable right $\Lambda$-module. Then the following statements hold.

(a) If $M$ is a 4-factor serial right $\Lambda$-module, then one of the following situations hold:

(i) $M$ is a local and colocal. Submodules of $M$ are $\operatorname{rad}(M)$ which is indecomposable non-local 4-factor serial, two indecomposable submodules $M_{1}$ and $M_{2}$ of length 3 that $M_{1}$ is uniserial and $M_{2}$ is non-local 3-factor serial, two indecomposable uniserial submodules $N_{1}$ and $N_{2}$ of length 2 which $N_{1}$ is a submodule of $M_{1}$ and $M_{2}$, and $N_{2}$ is a submodule of $M_{2}$ and $\operatorname{soc}(M)=S$ which is simple.

(ii) $M$ is local and non-colocal. Submodules of $M$ are $\operatorname{rad}(M)=N \oplus S_{1}$ that $N$ is uniserial of length 3 and $S_{1}$ is a simple right $\Lambda$-module, uniserial submodule $N_{1}$ of length 2 which is a submodule of $N$ and $\operatorname{soc}(M)=S_{1} \oplus S_{2}$ that $\operatorname{soc}(N)=S_{2}$.

(iii) $M$ is local and non-colocal. Submodules of $M$ are $\operatorname{rad}(M)=N_{1} \oplus N_{2}$ that for each $1 \leq i \leq 2, N_{i}$ is uniserial of length 2 and $\operatorname{soc}(M)=S_{1} \oplus S_{2}$ where for each $1 \leq i \leq 2, \operatorname{soc}\left(N_{i}\right)=S_{i}$.

(iv) $M$ is non-local of length 4 that soc $(M)$ is simple. Submodules of $M$ are two submodules $M_{1}$ and $M_{2}$ of length 3 that $M_{1}$ is uniserial and $M_{2}$ is non-local 3factor serial, two indecomposable uniserial submodules $N_{1}$ and $N_{2}$ of length 2 that $N_{1}$ is a submodule of $M_{1}$ and $M_{2}$ and $N_{2}$ is a submodule of $M_{2}$ and $\operatorname{soc}(M)=S$ which is simple.

(v) $M$ is non-local of length 4 that soc $(M)$ is non-simple. Submodules of $M$ are indecomposable modules $N_{1}$ of length 3 which is 2-factor serial, uniserial module $N_{2}$ of length 2 and $\operatorname{soc}(M)=S_{1} \oplus S_{2}$ where $S_{i}$ is a simple submodule of $M$ for each $1 \leq i \leq 2$ and $\operatorname{soc}\left(N_{2}\right)=S_{2}$.

(b) If $M$ is a 3-factor serial right $\Lambda$-module, then one of the following situations hold. 
(i) $M$ is local and colocal. Submodules of $M$ are $\operatorname{rad}(M)$ which is non-local 3-factor serial of length 3 , two uniserial submodules $N_{1}$ and $N_{2}$ of length 2 and $\operatorname{soc}(M)=S$ which is simple.

(ii) $M$ is local and $\operatorname{soc}(M)$ is not simple. Submodules of $M$ are $\operatorname{rad}(M)=N \oplus S_{1}$ that $N$ is uniserial of length $2, S_{1}$ is simple and $\operatorname{soc}(M)=S_{1} \oplus S_{2}$ that $\operatorname{soc}(N)=S_{2}$ which is simple.

(iii) $M$ is non-local of length 3. Submodules of $M$ are two uniserial submodules $N_{1}$ and $N_{2}$ of length 2 and $\operatorname{soc}(M)=S$ which is simple.

(c) If $M$ is a 2-factor serial local module, then submodules of $M$ are $\operatorname{rad}(M)=\operatorname{soc}(M)=$ $S_{1} \oplus S_{2}$ that $S_{1}$ and $S_{2}$ are simple right $\Lambda$-modules.

Proof. We prove only parts $(a)(i)$ and $(a)(v)$, the proof of the other parts is similar.

$(a)(i)$. Since $M$ is a local 4 -factor serial right $\Lambda$-module, by the Proposition $2.4, l(M)=5$ and it implies that $l(\operatorname{rad}(M))=4$. By [6, Theorem 2.6], $\operatorname{rad}(M)$ is 4-factor serial and so by [6, Corollary 2.8], $\operatorname{rad}(M)$ is non-local indecomposable. By the Proposition 3.2 , $l(\operatorname{top}(\operatorname{rad}(M)))=2$. Assume that $\operatorname{top}(\operatorname{rad}(M))=S_{1} \oplus S_{2}$, where $S_{1}$ and $S_{2}$ are simple $\Lambda$-modules. We claim that $\operatorname{rad}(M)$ has two maximal submodules. Since $\operatorname{rad}(M)$ is nonlocal, $\operatorname{rad}(M)$ has at least two maximal submodules $M_{1}$ and $M_{2}$ that $\frac{\operatorname{rad}(M)}{M_{1}} \cong S_{2}$ and $\frac{\operatorname{rad}(M)}{M_{2}} \cong S_{1}$. It implies that $S_{1}$ is a direct summand of $\operatorname{top}\left(M_{1}\right), S_{2}$ is a direct summand of top $\left(M_{2}\right)$ and we have an exact sequence

$$
0 \longrightarrow \operatorname{rad}^{2}(M) \longrightarrow \operatorname{rad}(M) \longrightarrow \frac{\operatorname{rad}(M)}{\operatorname{rad}^{2}(M)} \longrightarrow 0
$$

Hence $l\left(\operatorname{rad}^{2}(M)\right)=2 . \quad l(\operatorname{rad}(M))=4$ and the length of any maximal submodule of $\operatorname{rad}(M)$ is 3 , then $\operatorname{rad}(M)$ has two maximal submodules $M_{1}$ and $M_{2}$. Since for every $1 \leq i \leq 2, \operatorname{soc}(M)=\operatorname{soc}\left(M_{i}\right)$ which is simple, $M_{i}$ is indecomposable and by $[\underline{6}$, Theorem 5.2], $M_{1}$ and $M_{2}$ are not 2-factor serial. If $M_{i}$ is local, then $M_{i}$ is uniserial and if $M_{i}$ is non-local, then $M_{i}$ is 3 -factor serial for $1 \leq i \leq 2$. We show that $M_{1}$ is uniserial and $M_{2}$ is 3-factor serial. Assume that $S_{3}=\operatorname{top}\left(\operatorname{rad}^{2}(M)\right)$ and $S_{4}=\operatorname{soc}(M)$, where $S_{3}$ and $S_{4}$ are simple. If $M_{1}$ and $M_{2}$ are uniserial, then $\operatorname{top}\left(M_{1}\right)=S_{1}, \operatorname{top}\left(M_{2}\right)=S_{2}$ and $P_{1}, P_{2}$ are projective covers of $M_{1}, M_{2}$, respectively. Since $\operatorname{rad}^{2}(M) \subset M_{i}, S_{3}$ is a direct summand of $\operatorname{top}\left(\operatorname{rad}\left(P_{i}\right)\right)$ for each $1 \leq i \leq 2$. By [3, Proposition III.1.15], there are one arrow from the vertex 1 to the vertex 3 and one arrow from the vertex 2 to the vertex 3 in the valued quiver of $\Lambda$. Also $P_{3}$ is the projective cover of $\operatorname{rad}^{2}(M)$ and hence $S_{4}$ is a direct summand of $\operatorname{top}\left(\operatorname{rad}\left(P_{3}\right)\right)$. By [3, Proposition III.1.15], there is one arrow from the vertex 3 to the vertex 4 in the valued quiver of $\Lambda$. Then the valued quiver of $\Lambda$ has a subquiver of the form

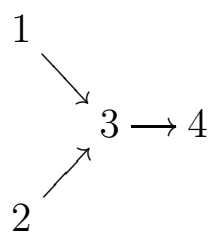

Therefore, there exists a non-local indecomposable right $\Lambda$-module $L$ of length 5 . By 6 , Corollary 2.8], $L$ is 5 -factor serial which gives a contradiction. Now assume that $M_{1}$ and $M_{2}$ are 3 -factor serial. Since $\frac{\operatorname{rad}(M)}{M_{1}} \cong S_{2}$ and $\frac{\operatorname{rad}(M)}{M_{2}} \cong S_{1}, S_{2}$ is not a direct summand 
of $\operatorname{top}\left(M_{1}\right)$ and $S_{1}$ is not a direct summand of $\operatorname{top}\left(M_{2}\right)$. Since $\operatorname{rad}\left(M_{i}\right) \subset M_{i}$ for every $1 \leq i \leq 2, \operatorname{top}\left(M_{1}\right)=S_{1} \oplus S_{3}$ and $\operatorname{top}\left(M_{2}\right)=S_{2} \oplus S_{3}$ and so $P_{1} \oplus P_{3}, P_{2} \oplus P_{3}$ are projective covers of $M_{1}$ and $M_{2}$, respectively. Then by [3, Proposition III.1.15], the valued quiver of $\Lambda$ has a subquiver of the form

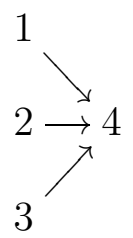

which gives a contradiction. So one of the maximal submodules $M_{1}$ and $M_{2}$ is uniserial and the other one is 3 -factor serial.

$(a)(v)$. Assume that $M$ is non-local of length 4 that $\operatorname{soc}(M)$ is not simple. By the Proposition [3.2, $l(\operatorname{top}(M))=2$ and it implies that $\operatorname{rad}(M)=\operatorname{soc}(M)=S_{3} \oplus S_{4}$ and $\operatorname{top}(M)=S_{1} \oplus S_{2}$, where $S_{i}$ is simple for each $i$. $M$ has two maximal submodules $M_{1}$ and $M_{2}$ of length 3 that $\operatorname{rad}(M)=\operatorname{soc}(M) \subset M_{i}$ for each $1 \leq i \leq 2, \frac{M}{M_{1}} \cong S_{2}, \frac{M}{M_{2}} \cong S_{1}$ and by [6. Lemma 5.2] if $M_{i}$ is indecomposable for each $1 \leq i \leq 2$, then $M_{i}$ is 2 -factor serial. We show that only one of the $M_{1}$ and $M_{2}$ is indecomposable. Assume on the contrary that, $M_{1}$ and $M_{1}$ are not indecomposable. $P_{1} \oplus P_{2}$ is a projective cover of $M$ and so $\frac{P_{1} \oplus P_{2}}{L} \cong M$, where $L$ is a submodule of $P_{1} \oplus P_{2}$ and $\frac{\operatorname{rad}\left(P_{1} \oplus P_{2}\right)}{L} \cong \operatorname{rad}(M) \cong \operatorname{soc}(M)=S_{3} \oplus S_{4}$. Therefore each $S_{i}, 3 \leq i \leq 4$ is a direct summand of one of the $\operatorname{top}\left(\operatorname{rad}\left(P_{1}\right)\right)$ and $\operatorname{top}\left(\operatorname{rad}\left(P_{2}\right)\right)$. Assume that $S_{3}$ is direct summand of $\operatorname{top}\left(\operatorname{rad}\left(P_{1}\right)\right)$ and $S_{4}$ is direct summand of top $\left(\operatorname{rad}\left(P_{2}\right)\right)$. Since $M_{1}$ and $M_{2}$ are not indecomposable, $S_{4}$ is not a direct summand of top $\left(\operatorname{rad}\left(P_{1}\right)\right)$ and $S_{3}$ is not a direct summand of $\operatorname{top}\left(\operatorname{rad}\left(P_{2}\right)\right)$. Then $M$ is a direct summand of two uniserial modules of length 2 which gives a contradiction. Now assume that $M_{1}$ and $M_{2}$ are both indecomposable 2 -factor serial right $\Lambda$-modules. Since for every $1 \leq i \leq 2$, $S_{1} \oplus S_{2}=\operatorname{rad}(M) \subset M_{i}$ and $P_{1}, P_{2}$ are projective covers of $M_{1}, M_{2}$, respectively. Then $S_{3}$ and $S_{4}$ are direct summands of $\operatorname{top}\left(\operatorname{rad}\left(P_{i}\right)\right)$ for each $1 \leq i \leq 2$ and by [3, Proposition III.1.15], the valued quiver of $\Lambda$ has a subquiver of the form

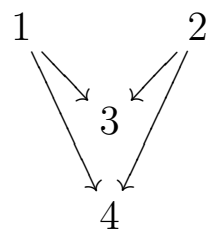

Therefore $\Lambda$ is not representation-finite which gives a contradiction. Then we can assume that $M_{1}$ is indecomposable and 2-factor serial and $M_{2}=N \oplus S$ that $N$ is uniserial of length 2 and $S$ is a simple right $\Lambda$-module.

Now by using the Theorem [3.3, we give a characterization of indecomposable nonprojective modules over right 4-Nakayama artin algebras.

Theorem 3.4. Let $\Lambda$ be a right 4-Nakayama artin algebra and $M$ be an indecomposable right $\Lambda$-module. Then $M$ is non-projective if and only if one of the following situations hold. 
(A) $M$ is a factor module of an indecomposable projective right $\Lambda$-module $P$ which is uniserial and $M \cong \frac{P}{\operatorname{rad}^{t}(P)}$ for some $1 \leq t<l(P)$.

(B) $M$ is a factor module of an indecomposable projective right $\Lambda$-module $P$ which is a 2-factor serial right $\Lambda$-module that $\operatorname{rad}(P)=\operatorname{soc}(P)=S_{1} \oplus S_{2}$ and $M$ is isomorphic to either $\frac{P}{\operatorname{rad}(P)}$ or $\frac{P}{S_{i}}$ for some $1 \leq i \leq 2$.

(C) $M$ is a factor module of an indecomposable projective right $\Lambda$-module $P$ which is a 3 -factor serial colocal right $\Lambda$-module such that submodules of $P$ are $\operatorname{rad}(P)$ which is 3-factor serial of length 3 and non-local, two uniserial submodules $N_{1}$ and $N_{2}$ of length 2 and $\operatorname{soc}(P)=S$ which is simple. $M$ is isomorphic to either $\frac{P}{\operatorname{rad}(P)}$ or $\frac{P}{N_{i}}$ for some $1 \leq i \leq 2$ or $\frac{P}{S}$.

(D) $M$ is a factor module of an indecomposable projective right $\Lambda$-module $P$ which is a 3-factor serial non-colocal right $\Lambda$-module such that submodules of $P$ are $\operatorname{rad}(P)=$ $N \oplus S_{1}$ that $N$ is uniserial of length $2, S_{1}$ is simple and $\operatorname{soc}(P)=S_{1} \oplus S_{2}$ that $\operatorname{soc}(N)=S_{2} . \quad M$ is isomorphic to either $\frac{P}{\operatorname{rad}(P)}$ or $\frac{P}{N}$ or $\frac{P}{\operatorname{soc}(P)}$ or $\frac{P}{S_{i}}$ for some $1 \leq i \leq 2$.

(E) $M$ is a factor module of an indecomposable projective right $\Lambda$-module $P$ which is colocal 4-factor serial right $\Lambda$-module such that submodules of $P$ are $\operatorname{rad}(P)$ which is 4-factor serial of length 4 and non-local, two submodules $M_{1}$ and $M_{2}$ of length 3 that $M_{1}$ is uniserial and $M_{2}$ is non-local 3-factor serial, two uniserial submodules $N_{1}$ and $N_{2}$ of length 2 that $N_{1}$ is a submodule of $M_{1}$ and $M_{2}$ and $N_{2}$ is a submodule of $M_{2}$ and $\operatorname{soc}(P)=S$ which is simple. $M$ is isomorphic to either $\frac{P}{\operatorname{rad}(P)}$ or $\frac{P}{M_{i}}$ for some $1 \leq i \leq 2$ or $\frac{P}{N_{i}}$ for some $1 \leq i \leq 2$ or $\frac{P}{S}$.

$(F) M$ is a factor module of an indecomposable projective right $\Lambda$-module $P$ which is a non-colocal 4-factor serial right $\Lambda$-module such that submodules of $P$ are $\operatorname{rad}(P)=$ $N \oplus S_{1}$ that $N$ is uniserial of length 3 and $S_{1}$ is a simple right $\Lambda$-module, an indecomposable uniserial submodule $N_{1}$ of length 2 and $\operatorname{soc}(P)=S_{1} \oplus S_{2}$ that $\operatorname{soc}(N)=S_{2}$. $M$ is isomorphic to either $\frac{P}{\operatorname{rad}(P)}$ or $\frac{P}{N}$ or $\frac{P}{N_{1}}$ or $\frac{P}{N_{1} \oplus S_{1}}$ or $\frac{P}{\operatorname{soc}(P)}$ or $\frac{P}{S_{i}}$ for some $1 \leq i \leq 2$.

(G) $M$ is a factor module of an indecomposable projective right $\Lambda$-module $P$ which is a non-colocal 4-factor serial right $\Lambda$-module such that submodules of $P$ are $\operatorname{rad}(P)=$ $N_{1} \oplus N_{2}$ that $N_{i}$ is uniserial of length 2 for each $1 \leq i \leq 2$ and $\operatorname{soc}(P)=S_{1} \oplus S_{2}$ that $\operatorname{soc}\left(N_{i}\right)=S_{i}$ for each $1 \leq i \leq 2$. $M$ is isomorphic to either $\frac{P}{\operatorname{rad}(P)}$ or $\frac{P}{N_{i}}$ for some $1 \leq i \leq 2$ or $\frac{P}{N_{1} \oplus S_{2}}$ or $\frac{P}{N_{2} \oplus S_{1}}$ or $\frac{P}{\operatorname{soc}(P)}$ or $\frac{P}{S_{i}}$ for some $1 \leq i \leq 2$.

(H) $M$ is a non-local 3-factor serial right $\Lambda$-module whose submodules are two uniserial modules $M_{1}$ and $M_{2}$ and $\operatorname{soc}(M)=S$ which is simple. Indecomposable quotients of $M$ are $\frac{M}{M_{i}}$ for $1 \leq i \leq 2$.

(I) $M$ is a non-local 4-factor serial right $\Lambda$-module with simple socle whose submodules are two maximal submodules $M_{1}$ and $M_{2}$ of length 3 that $M_{1}$ is uniserial and $M_{2}$ is non-local 3-factor serial, two uniserial submodules $N_{1}$ and $N_{2}$ of length 2 which $N_{1}$ is a uniserial submodule of $M_{1}$ and $M_{2}$ and $N_{2}$ is a submodule of $M_{2}$ and $\operatorname{soc}(M)=S$, which is simple. Indecomposable quotients of $M$ are $\frac{M}{M_{i}}$ for $1 \leq i \leq 2$ and $\frac{M}{N_{2}}$. 
A CHARACTERIZATION OF RIGHT 4-NAKAYAMA ARTIN ALGEBRAS

(J) $M$ is a non-local 4-factor serial right $\Lambda$-module that $\operatorname{soc}(M)$ is not simple. Submodules of $M$ are, an indecomposable module $N_{1}$ of length 3 which is 2-factor serial, a uniserial module $N_{2}$ of length 2 and $\operatorname{soc}(M)=S_{1} \oplus S_{2}$ that $\operatorname{soc}\left(N_{2}\right)=S_{2}$. Indecomposable quotients of $M$ are $\frac{M}{N_{i}}$ for $1 \leq i \leq 2, \frac{M}{N_{2} \oplus S_{1}}$ and $\frac{M}{S_{1}}$.

\section{Almost SPlit SEQUENCES OF RIGHT 4-NAKAyAmA ARTIN ALGEBRAS}

Now we compute almost split sequences of right 4-Nakayama artin algebras.

Theorem 4.1. Let $\Lambda$ be a right 4-Nakayama artin algebra and $M$ be an indecomposable non-projective right $\Lambda$-module. Then one of the following situations hold.

(A) Assume that $M \cong \frac{P}{\operatorname{rad}^{i}(P)}$, where $P$ is uniserial projective, $1 \leq i<l(P)$ and $M$ is not isomorphic to the quotient of an indecomposable non-local 3-factor serial module. Also assume that $M$ is not isomorphic to the either $\frac{L}{M_{1}}$ or $\frac{L}{N_{2}}$, where $L$ is an indecomposable colocal non-local 4-factor serial, $M_{1}$ is a uniserial submodule of $L$ and $N_{2}$ is a uniserial submodule of $L$ which is not a submodule of $M_{1}$. Then the following sequence is an almost split sequence.

$$
0 \longrightarrow \frac{\operatorname{rad}(P)}{\operatorname{rad}^{i+1}(P)} \stackrel{\left[\begin{array}{l}
\pi_{1} \\
i_{1}
\end{array}\right]}{\longrightarrow} \frac{\operatorname{rad}(P)}{\operatorname{rad}^{i}(P)} \oplus \frac{P}{\operatorname{rad}^{i+1}(P)} \stackrel{\left[-i_{2}, \pi_{2}\right]}{\longrightarrow} \frac{P}{\operatorname{rad}^{i}(P)} \longrightarrow 0
$$

(B) Assume that $M$ is a factor of an indecomposable projective 2-factor serial right $\Lambda$ module $P$. Submodules of $P$ are $\operatorname{rad}(P)=\operatorname{soc}(P)=S_{1} \oplus S_{2}$ that $S_{i}$ is a simple submodule of $P$ for each $1 \leq i \leq 2$. Also assume that $P$ is not a submodule of an indecomposable non-local 4-factor serial right $\Lambda$-module $L$ that $\operatorname{soc}(L)$ is not simple.

(i) If $M \cong \frac{P}{S_{i}}$ for some $1 \leq i \leq 2$, then the sequence

$$
0 \longrightarrow S_{i} \stackrel{i_{3}}{\longrightarrow} P \stackrel{\pi_{3}}{\longrightarrow} \frac{P}{S_{i}} \longrightarrow 0
$$

is an almost split sequence.

(ii) If $M \cong \frac{P}{\operatorname{rad}(P)}$, then the sequence

$$
0 \longrightarrow P \stackrel{\left[\begin{array}{l}
\pi_{4} \\
\pi_{5}
\end{array}\right]}{\longrightarrow} \frac{P}{S_{1}} \oplus \frac{P}{S_{2}} \stackrel{\left[-\pi_{6}, \pi_{7}\right]}{\longrightarrow} \frac{P}{\operatorname{rad}(P)} \longrightarrow 0
$$

is an almost split sequence.

$(C)$ Assume that $M$ is a factor of an indecomposable projective 3-factor serial colocal right $\Lambda$-module $P$. Submodules of $P$ are $\operatorname{rad}(P)$ which is indecomposable non-local 3-factor serial of length 3 , two uniserial modules $M_{1}$ and $M_{2}$ of length 2 and $S=\operatorname{soc}(P)$ which is simple.

(i) If $M \cong \frac{P}{\operatorname{rad}(P)}$, then the sequence

$$
0 \longrightarrow \frac{P}{S} \stackrel{\left[\begin{array}{l}
\pi_{8} \\
\pi_{9}
\end{array}\right]}{\longrightarrow} \frac{P}{M_{1}} \oplus \frac{P}{M_{2}} \stackrel{\left[-\pi_{10}, \pi_{11}\right]}{\longrightarrow} \frac{P}{\operatorname{rad}(P)} \longrightarrow 0
$$

is an almost split sequence.

(ii) If $M \cong \frac{P}{M_{i}}$, for some $1 \leq i \leq 2$, then the sequence 


$$
0 \longrightarrow \frac{M_{i}}{S} \stackrel{i_{4}}{\longrightarrow} \frac{P}{S} \stackrel{\pi_{12}}{\longrightarrow} \frac{P}{M_{i}} \longrightarrow 0
$$

is an almost split sequence.

(iii) If $M \cong \frac{P}{S}$, then the sequence

$$
0 \longrightarrow \operatorname{rad}(P) \stackrel{\left[\begin{array}{c}
\pi_{13} \\
\pi_{14} \\
i_{5}
\end{array}\right]}{\longrightarrow} \frac{M_{1}}{S} \oplus \frac{M_{2}}{S} \oplus P \stackrel{\left[-i_{6}, i_{7}, \pi_{15}\right]}{\longrightarrow} \frac{P}{S} \longrightarrow 0
$$

is an almost split sequence.

(D) Assume that $M$ is a factor of an indecomposable projective 3-factor serial non-colocal right $\Lambda$-module $P$. Submodules of $P$ are $\operatorname{rad}(P)=N \oplus S_{1}$ that $N$ is uniserial of length 2, $S_{1}$ is simple and $\operatorname{soc}(P)=S_{1} \oplus S_{2}$ that $\operatorname{soc}(N)=S_{2}$.

(i) If $M \cong \frac{P}{\operatorname{rad}(P)}$, then the sequence

$$
0 \longrightarrow \frac{P}{S_{2}} \stackrel{\left[\begin{array}{l}
\pi_{16} \\
\pi_{17}
\end{array}\right]}{\longrightarrow} \frac{P}{N} \oplus \frac{P}{\operatorname{soc}(P)} \stackrel{\left[-\pi_{18}, \pi_{19}\right]}{\longrightarrow} \frac{P}{\operatorname{rad}(P)} \longrightarrow 0
$$

is an almost split sequence.

(ii) If $M \cong \frac{P}{N}$, then the sequence

$$
0 \longrightarrow \frac{N}{S_{2}} \stackrel{i_{8}}{\longrightarrow} \frac{P}{S_{2}} \stackrel{\pi_{20}}{\longrightarrow} \frac{P}{N} \longrightarrow 0
$$

is an almost split sequence.

(iii) If $M \cong \frac{P}{\operatorname{soc}(P)}$, then the sequence

$$
0 \longrightarrow P \stackrel{\left[\begin{array}{l}
\pi_{21} \\
\pi_{22}
\end{array}\right]}{\longrightarrow} \frac{P}{S_{1}} \oplus \frac{P}{S_{2}} \stackrel{\left[-\pi_{23}, \pi_{24}\right]}{\longrightarrow} \frac{P}{\operatorname{soc}(P)} \longrightarrow 0
$$

is an almost split sequence.

(iv) If $M \cong \frac{P}{S_{1}}$, then the sequence

$$
0 \longrightarrow S_{1} \stackrel{i_{9}}{\longrightarrow} P \stackrel{\pi_{25}}{\longrightarrow} \frac{P}{S_{1}} \longrightarrow 0
$$

is an almost split sequence.

(v) If $M \cong \frac{P}{S_{2}}$, then the sequence

$$
0 \longrightarrow N \stackrel{\left[\begin{array}{c}
\pi_{26} \\
i_{10}
\end{array}\right]}{\longrightarrow} \frac{N}{S_{2}} \oplus P \stackrel{\left[-i_{11}, \pi_{27}\right]}{\longrightarrow} \frac{P}{S_{2}} \longrightarrow 0
$$

is an almost split sequence.

$(E)$ Assume that $M$ is a factor of an indecomposable projective colocal 4-factor serial right $\Lambda$-module $P$. Submodules of $P$ are $\operatorname{rad}(P)$ that is non-local 4-factor serial of length 4, two submodules $M_{1}$ and $M_{2}$ of length 3 that $M_{1}$ is uniserial and $M_{2}$ is non-local 3-factor serial, two uniserial submodules $N_{1}$ and $N_{2}$ of length 2 that $N_{1}$ is a submodule of $M_{1}$ and $M_{2}, N_{2}$ is a submodule of $M_{2}$ and $\operatorname{soc}(P)=S$ which is simple.

(i) If $M \cong \frac{P}{\operatorname{rad}(P)}$, then the sequence 


$$
0 \longrightarrow \frac{P}{N_{1}} \stackrel{\left[\begin{array}{l}
\pi_{28} \\
\pi_{29}
\end{array}\right]}{\longrightarrow} \frac{P}{M_{1}} \oplus \frac{P}{M_{2}} \stackrel{\left[-\pi_{30}, \pi_{31}\right]}{\longrightarrow} \frac{P}{\operatorname{rad}(P)} \longrightarrow 0
$$

is an almost split sequence.

(ii) If $M \cong \frac{P}{M_{2}}$, then the sequence

is an almost split sequence.

$$
0 \longrightarrow \frac{P}{S} \stackrel{\left[\begin{array}{l}
\pi_{32} \\
\pi_{33}
\end{array}\right]}{\longrightarrow} \frac{P}{N_{1}} \oplus \frac{P}{N_{2}} \stackrel{\left[-\pi_{34}, \pi_{35}\right]}{\longrightarrow} \frac{P}{M_{2}} \longrightarrow 0
$$

(iii) If $M \cong \frac{P}{M_{1}}$, then the sequence

$$
0 \longrightarrow \frac{M_{1}}{N_{1}} \stackrel{i_{12}}{\longrightarrow} \frac{P}{N_{1}} \stackrel{\pi_{36}}{\longrightarrow} \frac{P}{M_{1}} \longrightarrow 0
$$

is an almost split sequence.

(iv) If $M \cong \frac{P}{N_{1}}$, then the sequence

$$
0 \longrightarrow \frac{M_{1}}{S} \stackrel{\left[\begin{array}{c}
\pi_{37} \\
i_{13}
\end{array}\right]}{\longrightarrow} \frac{M_{1}}{N_{1}} \oplus \frac{P}{S} \stackrel{\left[-i_{14}, \pi_{38}\right]}{\longrightarrow} \frac{P}{N_{1}} \longrightarrow 0
$$

is an almost split sequence.

(v) If $M \cong \frac{P}{N_{2}}$, then the sequence

is an almost split sequence.

$$
0 \longrightarrow \frac{N_{2}}{S} \stackrel{i_{15}}{\longrightarrow} \frac{P}{S} \stackrel{\pi_{39}}{\longrightarrow} \frac{P}{N_{2}} \longrightarrow 0
$$

(vi) If $M \cong \frac{P}{S}$, then the sequence

$$
0 \longrightarrow \operatorname{rad}(P) \stackrel{\left[\begin{array}{l}
i_{16} \\
\pi_{40} \\
\pi_{41}
\end{array}\right]}{\longrightarrow} P \oplus \frac{M_{1}}{S} \oplus \frac{N_{2}}{S} \stackrel{\left[\pi_{42},-i_{17}, i_{18}\right]}{\longrightarrow} \frac{P}{S} \longrightarrow 0
$$

is an almost split sequence.

$(F)$ Assume that $M$ is a factor of an indecomposable projective non-colocal 4-factor serial right $\Lambda$-module $P$. Submodules of $P$ are $\operatorname{rad}(P)=N \oplus S_{1}$ that $N$ is a uniserial module of length 3 and $S_{1}$ is a simple right $\Lambda$-module, an indecomposable uniserial submodule $N_{1}$ of length 2 and $\operatorname{soc}(P)=S_{1} \oplus S_{2}$ that $S_{2}=\operatorname{soc}(N)$.

(i) If $M \cong \frac{P}{\operatorname{rad}(P)}$, then the sequence

$$
0 \longrightarrow \frac{P}{N_{1}} \stackrel{\left[\begin{array}{l}
\pi_{43} \\
\pi_{44}
\end{array}\right]}{\longrightarrow} \frac{P}{N_{1} \oplus S_{1}} \oplus \frac{P}{N} \stackrel{\left[-\pi_{45}, \pi_{46}\right]}{\longrightarrow} \frac{P}{\operatorname{rad}(P)} \longrightarrow 0
$$

is an almost split sequence.

(ii) If $M \cong \frac{P}{N}$, then the sequence

is an almost split sequence.

$$
0 \longrightarrow \frac{N}{N_{1}} \stackrel{i_{19}}{\longrightarrow} \frac{P}{N_{1}} \stackrel{\pi_{47}}{\longrightarrow} \frac{P}{N} \longrightarrow 0
$$

(iii) If $M \cong \frac{P}{N_{1} \oplus S_{1}}$, then the sequence 
is an almost split sequence.

$$
0 \longrightarrow \frac{P}{S_{2}} \stackrel{\left[\begin{array}{l}
\pi_{48} \\
\pi_{49}
\end{array}\right]}{\longrightarrow} \frac{P}{\operatorname{soc}(P)} \oplus \frac{P}{N_{1}} \stackrel{\left[-\pi_{50}, \pi_{51}\right]}{\longrightarrow} \frac{P}{N_{1} \oplus S_{1}} \longrightarrow 0
$$

(iv) If $M \cong \frac{P}{\operatorname{soc}(P)}$, then the sequence

is an almost split sequence.

$$
0 \longrightarrow P \stackrel{\left[\begin{array}{l}
\pi_{52} \\
\pi_{53}
\end{array}\right]}{\longrightarrow} \frac{P}{S_{1}} \oplus \frac{P}{S_{2}} \stackrel{\left[-\pi_{54, \pi}\right.}{\longrightarrow} \frac{P}{\operatorname{soc}(P)} \longrightarrow 0
$$

(v) If $M \cong \frac{P}{N_{1}}$, then the sequence

is an almost split sequence.

$$
0 \longrightarrow \frac{N}{S_{2}} \stackrel{\left[\begin{array}{l}
i_{20} \\
\pi_{56}
\end{array}\right]}{\longrightarrow} \frac{P}{S_{2}} \oplus \frac{N}{N_{1}} \stackrel{\left[-\pi_{57}, i_{21}\right]}{\longrightarrow} \frac{P}{N_{1}} \longrightarrow 0
$$

(vi) If $M \cong \frac{P}{S_{1}}$, then the sequence

$$
0 \longrightarrow S_{1} \stackrel{i_{22}}{\longrightarrow} P \stackrel{\pi_{58}}{\longrightarrow} \frac{P}{S_{1}} \longrightarrow 0
$$

is an almost split sequence.

(vii) If $M \cong \frac{P}{S_{2}}$, then the sequence

$$
0 \longrightarrow N \stackrel{\left[\begin{array}{l}
i_{23} \\
\pi_{59}
\end{array}\right]}{\longrightarrow} P \oplus \frac{N}{S_{2}} \stackrel{\left[-\pi_{60}, i_{24}\right]}{\longrightarrow} \frac{P}{S_{2}} \longrightarrow 0
$$

is an almost split sequence.

$(G)$ Assume that $M$ is a factor of an indecomposable projective non-colocal 4-factor serial right $\Lambda$-module $P$. Submodules of $P$ are $\operatorname{rad}(P)=N_{1} \oplus N_{2}$ that for each $1 \leq i \leq 2, N_{i}$ is uniserial of length 2 and $\operatorname{soc}(P)=S_{1} \oplus S_{2}$ that for each $1 \leq i \leq 2, \operatorname{soc}\left(N_{i}\right)=S_{i}$.

(i) If $M \cong \frac{P}{\operatorname{rad}(P)}$, then the sequence

$$
0 \longrightarrow \frac{P}{\operatorname{soc}(P)} \stackrel{\left[\begin{array}{l}
\pi_{61} \\
\pi_{62}
\end{array}\right]}{\longrightarrow} \frac{P}{N_{1} \oplus S_{2}} \oplus \frac{P}{N_{2} \oplus S_{1}} \stackrel{\left[-\pi_{63}, \pi_{64}\right]}{\longrightarrow} \frac{P}{\operatorname{rad}(P)} \longrightarrow 0
$$

is an almost split sequence.

(ii) If $M \cong \frac{P}{N_{i} \oplus S_{j}}$ for some $1 \leq i, j \leq 2$ and $i \neq j$, then the sequence

$$
0 \longrightarrow \frac{P}{S_{i}} \stackrel{\left[\begin{array}{l}
\pi_{65} \\
\pi_{66}
\end{array}\right]}{\longrightarrow} \frac{P}{\operatorname{soc}(P)} \oplus \frac{P}{N_{i}} \stackrel{\left[-\pi_{67}, \pi_{68}\right]}{\longrightarrow} \frac{P}{N_{i} \oplus S_{j}} \longrightarrow 0
$$

is an almost split sequence.

(iii) If $M \cong \frac{P}{N_{i}}$ for some $1 \leq i \leq 2$, then the sequence

$$
0 \longrightarrow \frac{N_{i}}{S_{i}} \stackrel{i_{25}}{\longrightarrow} \frac{P}{S_{i}} \stackrel{\pi_{69}}{\longrightarrow} \frac{P}{N_{i}} \longrightarrow 0
$$

is an almost split sequence.

(iv) If $M \cong \frac{P}{\operatorname{soc}(P)}$, then the sequence 


$$
0 \longrightarrow P \stackrel{\left[\begin{array}{l}
\pi_{70} \\
\pi_{71}
\end{array}\right]}{\longrightarrow} \frac{P}{S_{1}} \oplus \frac{P}{S_{2}} \stackrel{\left[-\pi_{72}, \pi_{73}\right]}{\longrightarrow} \frac{P}{\operatorname{soc}(P)} \longrightarrow 0
$$

is an almost split sequence.

(v) If $M \cong \frac{P}{S_{i}}$ for some $1 \leq i \leq 2$, then the sequence

$$
0 \longrightarrow N_{i} \stackrel{\left[\begin{array}{l}
\pi_{74} \\
i_{26}
\end{array}\right]}{\longrightarrow} \frac{N_{i}}{S_{i}} \oplus P \stackrel{\left[-i_{27}, \pi_{75}\right]}{\longrightarrow} \frac{P}{S_{i}} \longrightarrow 0
$$

is an almost split sequence.

(H) Assume that $M$ is an indecomposable non-local 3-factor serial right $\Lambda$-module which is not isomorphic to the quotient of an indecomposable non-local non-colocal 4-factor serial right $\Lambda$-module $L . M$ is of length 3 and submodules of $M$ are two uniserial maximal submodules $M_{1}$ and $M_{2}$ of length 2 and $\operatorname{rad}(M)=\operatorname{soc}(M)=S$ which is simple.

(i) The exact sequence

$$
0 \longrightarrow S \stackrel{\left[\begin{array}{l}
i_{29} \\
i_{30}
\end{array}\right]}{\longrightarrow} M_{1} \oplus M_{2} \stackrel{\left[-i_{31}, i_{32}\right]}{\longrightarrow} M \longrightarrow 0
$$

is an almost split sequence.

(ii) For an indecomposable right $\Lambda$-module $\frac{M}{M_{j}}, 1 \leq j \leq 2$, the exact sequence

$$
0 \longrightarrow M_{j} \stackrel{i_{28}}{\longrightarrow} M \stackrel{\pi_{76}}{\longrightarrow} \frac{M}{M_{j}} \longrightarrow 0
$$

is an almost split sequence.

(I) Assume that $M$ is an indecomposable non-local 4-factor serial right $\Lambda$-module that $\operatorname{soc}(M)$ is simple. Submodules of $M$ are two indecomposable submodules $M_{1}$ and $M_{2}$ of length 3 that $M_{1}$ is uniserial and $M_{2}$ is non-local, two uniserial submodules $N_{1}$ and $N_{2}$ of length 2 that $N_{1}$ is a submodule of $M_{1}$ and $M_{2}, N_{2}$ is a submodule of $M_{2}$ and $\operatorname{soc}(M)=S$ which is simple.

(i) The exact sequence

$$
0 \longrightarrow N_{1} \stackrel{\left[\begin{array}{l}
i_{33} \\
i_{34}
\end{array}\right]}{\longrightarrow} M_{1} \oplus M_{2} \stackrel{\left[-i_{35}, i_{36}\right]}{\longrightarrow} M \longrightarrow 0
$$

is an almost split sequence.

(ii) For an indecomposable module $\frac{M}{M_{1}}$, the exact sequence

$$
0 \longrightarrow M_{1} \stackrel{i_{37}}{\longrightarrow} M \stackrel{\pi_{77}}{\longrightarrow} \frac{M}{M_{1}} \longrightarrow 0
$$

is an almost split sequence.

(iii) For an indecomposable module $\frac{M}{N_{2}}$, the exact sequence

$$
0 \longrightarrow M_{2} \stackrel{\left[\begin{array}{c}
\pi_{78} \\
i_{38}
\end{array}\right]}{\longrightarrow} \operatorname{soc}\left(\frac{M}{N_{2}}\right) \oplus M \stackrel{\left[-i_{39}, \pi_{79}\right]}{\longrightarrow} \frac{M}{N_{2}} \longrightarrow 0
$$

is an almost split sequence. 
( $J)$ Assume that $M$ is an indecomposable non-local 4-factor serial right $\Lambda$-module of length 4 that $\operatorname{soc}(M)$ is not simple. Submodules of $M$ are $M_{1}$ and $M_{2}$ that $M_{1}$ is 2-factor serial of length $3, M_{2}$ is uniserial of length 2 and $\operatorname{soc}(M)=S_{1} \oplus S_{2}$ that $\operatorname{soc}\left(M_{2}\right)=S_{2}$.

(i) The sequence

$$
0 \longrightarrow S_{2} \stackrel{\left[\begin{array}{c}
\pi_{80} \\
i_{40}
\end{array}\right]}{\longrightarrow} M_{1} \oplus M_{2} \stackrel{\left[-i_{41}, \pi_{81}\right]}{\longrightarrow} M \longrightarrow 0
$$

is an almost split sequence.

(ii) For an indecomposable module $\frac{M}{S_{1}}$ the sequence

$$
0 \longrightarrow M_{1} \stackrel{\left[\begin{array}{c}
\pi_{82} \\
i_{42}
\end{array}\right]}{\longrightarrow} \frac{M_{1}}{S_{1}} \oplus M \stackrel{\left[-i_{43}, \pi_{83}\right]}{\longrightarrow} \frac{M}{S_{1}} \longrightarrow 0
$$

is an almost split sequence.

(iii) For an indecomposable module $\frac{M}{M_{2}}$, the sequence

$$
0 \longrightarrow M_{2} \stackrel{i_{44}}{\longrightarrow} M \stackrel{\pi_{84}}{\longrightarrow} \frac{M}{M_{2}} \longrightarrow 0
$$

is an almost split sequence.

(iv) For an indecomposable module $\frac{M}{M_{2} \oplus S_{1}}$, the sequence

$$
0 \longrightarrow M \stackrel{\left[\begin{array}{l}
\pi_{85} \\
\pi_{86}
\end{array}\right]}{\longrightarrow} \frac{M}{S_{1}} \oplus \frac{M}{M_{2}} \stackrel{\left[-\pi_{87}, \pi_{88}\right]}{\longrightarrow} \frac{M}{M_{2} \oplus S_{1}} \longrightarrow 0
$$

is an almost split sequence.

(v) For an indecomposable module $\frac{M}{M_{1}}$, the sequence

$$
0 \longrightarrow \frac{M_{1}}{S_{1}} \stackrel{i_{45}}{\longrightarrow} \frac{M}{S_{1}} \stackrel{\pi_{89}}{\longrightarrow} \frac{M}{M_{1}} \longrightarrow 0
$$

is an almost split sequence.

Where $i_{j}$ is an inclusion for each $1 \leq j \leq 45$ and $\pi_{j}$ is a canonical epimorphism for each $1 \leq j \leq 89$.

Proof. We only prove the parts $(H)(i),(H)(i i),(I)(i),(I)(i i),(I)(i i i),(J)(i),(J)(i i)$, $(J)(i i i),(J)(i v)$ and $(J)(v)$. In the other parts, since $M$ is a quotient of an indecomposable projective right $\Lambda$-module without any condition, the proof is easy. Put $g_{1}=\left[-i_{31}, i_{32}\right]$, $g_{2}=\left[-i_{35}, i_{36}\right], g_{3}=\left[-i_{39}, \pi_{79}\right], g_{4}=\left[-i_{41}, \pi_{81}\right], g_{5}=\left[-i_{43}, \pi_{83}\right]$ and $g_{6}=\left[-\pi_{87}, \pi_{88}\right]$. It is easy to see that all given sequences are exact, non-split and have indecomposable end terms. Then it is enough to show that homomorphisms $g_{1}, \pi_{76}, g_{2}, \pi_{77}, g_{3}, g_{4}, g_{5}, \pi_{84}, g_{6}$ and $\pi_{89}$ are right almost split morphisms.

$(H)(i)$. Let $V$ be an indecomposable right $\Lambda$-module and $\nu: V \longrightarrow M$ be a non-isomorphism. If $\nu$ is an epimorphism, then by [6, Lemma 2.11], $V$ is a 4 -factor serial right $\Lambda$-module and by the Theorem $3.4 V$ is non-local non-colocal 4-factor serial. Then $M$ isomorphic to the quotient of an indecomposable non-local non-colocal 4-factor serial right $\Lambda$-module which is a contradiction. Thus $\operatorname{Im}(\nu)$ is a proper submodule of $M$. Since $M_{1}$ and $M_{2}$ 
are maximal submodules of $M, \operatorname{Im}(\nu)$ is a submodule of $M_{1} \oplus M_{2}$. Then there exists a homomorphism $h: V \longrightarrow M_{1} \oplus M_{2}$ such that $\nu=g_{1} h$.

$(H)(i i)$. Let $V$ be an indecomposable right $\Lambda$-module and $\nu: V \longrightarrow \frac{M}{M_{j}}$ be a nonisomorphism. Since $\frac{M}{M_{j}}$ is a simple right $\Lambda$-module, $\nu$ is an epimorphism. If $j=1$, then $V$ is isomorphic to either $M$ or $M_{2}$ or a quotient of an indecomposable projective right $\Lambda$-module $P$. If $V$ is isomorphic to either $M$ or $M_{2}$, then there exists a homomorphism $h: V \longrightarrow M$ such that $\pi_{76} h=\nu$. Now assume that $V$ is isomorphic to the quotient of $P$. We show that in this case $P$ is uniserial. Let $\frac{M}{M_{1}} \cong S_{2}, \operatorname{top}(M)=S_{1} \oplus S_{2}$ and $\operatorname{rad}(M)=\operatorname{soc}(M)=S_{3}$, then $P_{1} \oplus P_{2}$ is a projective cover of $M$ and $P=P_{2}$. By [3, Proposition III.1.15], the valued quiver of $\Lambda$ has a subquiver of the form

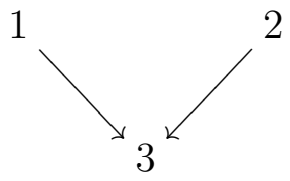

If $P_{2}$ is not uniserial, then by Theorem 3.3, $\operatorname{top}\left(\operatorname{rad}\left(P_{2}\right)\right)=S_{3} \oplus S_{4}$ and by [3, Proposition III.1.15], the valued quiver of $\Lambda$ has a subquiver of the form

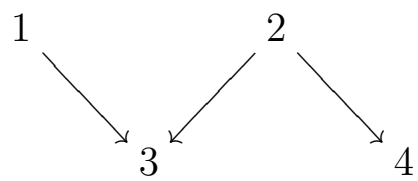

Thus $M$ is isomorphic to the quotient of an indecomposable non-local non-colocal 4 -factor serial right $\Lambda$-module $L$, which gives a contradiction. Then $P$ is uniserial. Also $M_{2}$ is a submodule of $M$ and $\operatorname{top}\left(\frac{P}{\operatorname{rad}^{i}(P)}\right)$ is direct summand of $\operatorname{top}(M)$ for some $2 \leq i<l(P)$. Therefore there exists a homomorphism $h: V \longrightarrow M$ such that $\pi_{76} h=\nu$.

$(I)(i)$. The proof is similar to the proof of $(H)(i)$.

$(I)(i i)$. Let $V$ be an indecomposable right $\Lambda$-module and $\nu: V \longrightarrow \frac{M}{M_{1}}$ be a non-isomorphism. Since $\frac{M}{M_{1}}$ is simple, $V$ is isomorphic to either $M$ or $M_{2}$ or $N_{2}$ or a quotient of an indecomposable projective right $\Lambda$-module $P$. The similar argument as in the proof of $(H)(i i)$ shows that $P$ is uniserial. Then there exists a homomorphism $h: V \longrightarrow M$ such that $\pi_{77} h=\nu$.

$(I)($ iii $)$. Let $V$ be an indecomposable right $\Lambda$-module and $\nu: V \longrightarrow \frac{M}{N_{2}}$ be non-isomorphism. If $\nu$ is an epimorphism, then $V$ is isomorphic to either $M$ or $M_{1}$ or a quotient of an indecomposable projective right $\Lambda$-module $P$. The similar argument as in the proof of $(H)(i i)$ shows that $P$ is uniserial. Since $\operatorname{top}\left(\frac{P}{\operatorname{rad}^{i}(P)}\right) \cong \operatorname{top}\left(\frac{M}{N_{2}}\right)$, there exists a homomorphism $h: V \longrightarrow \operatorname{soc}\left(\frac{M}{N_{2}}\right) \oplus M$ such that $g_{3} h=\nu$. If $\nu$ is not an epimorphism, then $\operatorname{Im} \nu=\operatorname{soc}\left(\frac{M}{N_{2}}\right)$ and so there exists a homomorphism $h: V \longrightarrow \operatorname{soc}\left(\frac{M}{N_{2}}\right) \oplus M$ such that $g_{3} h=\nu$.

$(J)(i)$. The proof is similar to the proof of $(H)(i)$.

$(J)(i i)$. Let $V$ be an indecomposable right $\Lambda$-module and $\nu: V \longrightarrow \frac{M}{S_{1}}$ be a non-isomorphism.

If $\nu$ is an epimorphism, then $V \cong M$ and there exists a homomorphism $h: V \longrightarrow \frac{M_{1}}{s_{1}} \oplus M$ such that $g_{5} h=\nu$. Now assume that $\nu$ is not an epimorphism. Maximal submodules 
of $\frac{M}{S_{1}}$ are $M_{2}$ and $\frac{M_{1}}{S_{1}}$, then there exists a homomorphism $h: V \longrightarrow \frac{M_{1}}{s_{1}} \oplus M$ such that $g_{5} h=\nu$.

$(J)($ iii $)$. Let $V$ be an indecomposable right $\Lambda$-module and $\nu: V \longrightarrow \frac{M}{M_{2}}$ be a nonisomorphism. If $\nu$ is an epimorphism, then $V$ is isomorphic to either $M$ or $M_{1}$ or a quotient of an indecomposable projective right $\Lambda$-module $P$. The similar argument as in the proof of $(H)(i i)$ shows that $M_{1}$ is a quotient of $P$. Then there exists a homomorphism $h: V \longrightarrow M$ such that $\pi_{84} h=\nu$. Now assume that $\nu$ is not an epimorphism. Since $\operatorname{Im}(\nu)=\operatorname{soc}\left(\frac{M}{M_{2}}\right) \subset \operatorname{soc}(M), \operatorname{Im}(\nu)$ is a submodule of $M$ and so there exists a homomorphism $h: V \longrightarrow M$ such that $\pi_{84} h=\nu$.

$(J)(i v)$. Let $V$ be an indecomposable right $\Lambda$-module and $\nu: V \longrightarrow \frac{M}{M_{2} \oplus S_{1}}$ be a nonisomorphism. Since $\frac{M}{M_{2} \oplus S_{1}}$ is simple, $V$ is isomorphic to either $M$ or $M_{1}$ or $\frac{M}{S_{1}}$ or $\frac{M}{M_{2}}$ or a quotient of an indecomposable projective right $\Lambda$-module $P$. The similar argument as in the proof of $(H)(i i)$ shows that $M_{1}$ is a quotient of $P$. then there exists a homomorphism $h: V \longrightarrow \frac{M}{S_{1}} \oplus \frac{M}{M_{2}}$ such that $g_{6} h=\nu$.

$(J)(v)$. The proof is similar to the proof of $(J)(i i i)$.

\section{QUIVERS AND RELATIONS OF RIGHT 4-NAKAYAMA FINITE DIMENSIONAL $K$-ALGEBRAS}

Let $\Lambda$ be a basic connected finite dimensional $K$-algebra. It is known that there exist a quiver $Q$ and an admissible ideal $I$ of the path algebra $K Q$ such that $\Lambda \cong \frac{K Q}{I}$. In this section, we give a necessary and sufficient conditions for the quiver $Q$ and the admissible ideal $I$ that $\frac{K Q}{I}$ be a right 4 -Nakayama algebra.

A finite dimensional $K$-algebra $\Lambda=\frac{K Q}{I}$ is called special biserial algebra provided $(Q, I)$ satisfying the following conditions:

(1) For any vertex $a \in Q_{0},\left|a^{+}\right| \leq 2$ and $\left|a^{-}\right| \leq 2$.

(2) For any arrow $\alpha \in Q_{1}$, there is at most one arrow $\beta$ and at most one arrow $\gamma$ such that $\alpha \beta$ and $\gamma \alpha$ are not in $I$.

Let $\Lambda=\frac{K Q}{I}$ be a special biserial finite dimensional $K$-algebra. A walk $w=c_{1} c_{2} \cdots c_{n}$ is called string of length $n$ if $c_{i} \neq c_{i+1}^{-1}$ for each $i$ and no subwalk nor its inverse is in $I$. In addition, we have strings of length zero, for any $a \in Q_{0}$ we have two strings of length zero, denoted by $1_{(a, 1)}$ and $1_{(a,-1)}$. We have $s\left(1_{(a, 1)}\right)=t\left(1_{(a, 1)}\right)=s\left(1_{(a,-1)}\right)=t\left(1_{(a,-1)}\right)=a$ and $1_{(a, 1)}^{-1}=1_{(a,-1)}$. A string $w=c_{1} c_{2} \cdots c_{n}$ with $s(w)=t(w)$ such that each power $w^{m}$ is a string, but $w$ itself is not a proper power of any strings is called band. We denote by $\mathcal{S}(\Lambda)$ and $\mathcal{B}(\Lambda)$ the set of all strings of $\Lambda$ and the set of all bands of $\Lambda$, respectively. Let $\rho$ be the equivalence relation on $\mathcal{S}(\Lambda)$ which identifies every string $w$ with its inverse $w^{-1}$ and $\sigma$ be the equivalence relation on $\mathcal{B}(\Lambda)$ which identifies every band $w=c_{1} c_{2} \cdots c_{n}$ with the cyclically permuted bands $w_{(i)}=c_{i} c_{i+1} \cdots c_{n} c_{1} \cdots c_{i-1}$ and their inverses $w_{(i)}^{-1}$, for each $i$. Butler and Ringle in [4] for each string $w$ defined a unique string module $M(w)$ and for each band $v$ defined a family of band modules $M(v, m, \varphi)$ with $m \geq 1$ and $\varphi \in A u t\left(K^{m}\right)$. Let $\widetilde{\mathcal{S}}(\Lambda)$ be the complete set of representatives of strings relative to $\rho$ and $\widetilde{\mathcal{B}}(\Lambda)$ be the complete set of representatives of bands relative to $\sigma$. Butler and Ringle in [4] proved that, the modules $M(w), w \in \widetilde{\mathcal{S}}(\Lambda)$ and the modules $M(v, m, \varphi)$ with 
$v \in \widetilde{\mathcal{B}}(\Lambda), m \geq 1$ and $\varphi \in A u t\left(K^{m}\right)$ provide complete list of pairwise non-isomorphic indecomposable $\Lambda$-modules. Indecomposable right $\Lambda$-modules are either string modules or band modules or non-uniserial projective-injective modules (see [4] and [10]). If $\Lambda$ is a representation-finite special biserial algebra, then any indecomposable right $\Lambda$-module is either string or non-uniserial projective-injective.

Proposition 5.1. Any basic connected finite dimensional right 4-Nakayama $K$-algebra is representation-finite special biserial.

Proof. The proof is similar to the proof of [5, Proposition 3.2].

Theorem 5.2. Let $\Lambda=\frac{K Q}{I}$ be a basic, connected and finite dimensional $K$-algebra. Then $\Lambda$ is a right 4-Nakayama algebra if and only if $\Lambda$ is a representation-finite special biserial algebra that $(Q, I)$ satisfying the following conditions:

(i) If there exist a walk $W$ and two different arrows $w_{1}$ and $w_{2}$ with the same target such that $w_{1}^{+1} w_{2}^{-1}$ is a subwalk of $W$, then length $(W) \leq 3$.

(ii) If there exist a walk $W$ and two different arrows $w_{1}$ and $w_{2}$ with the same source such that $w_{1}^{-1} w_{2}^{+1}$ is a subwalk of $W$, then length $(W) \leq 4$.

(iii) If there exist two paths $p$ and $q$ with the same target and the same source such that $p-q \in I$. Then length $(p)+$ length $(q) \leq 5$.

(iv) At least one of the following conditions hold:

(a) There exist a walk $W$ of length 3 and two different arrows $w_{1}$ and $w_{2}$ with the same target such that $w_{1}^{+1} w_{2}^{-1}$ is a subwalk of $W$.

(b) There exist a walk $W$ of length 4 and two different arrows $w_{1}$ and $w_{2}$ with the same source such that $w_{1}^{-1} w_{2}^{+1}$ is a subwalk of $W$.

(c) There exist two paths $p$ and $q$ with the same target and the same source such that $p-q \in I$ and length $(p)+$ length $(q)=5$.

Proof. Let $\Lambda$ be a right 4-Nakayama algebra. By Proposition 5.1, $\Lambda$ is a special biserial algebra of finite type. Assume on the contrary that the condition $(i)$ does not hold. Then there exists a walk $W$ of length greater than or equal 4 which has a subwalk $W^{\prime}$ of length 3 that $W^{\prime}$ has a subwalk of the form $w_{1}^{+1} w_{2}^{-1}$. Since $\Lambda$ is a special biserial algebra of finite type, the walk $W^{\prime}$ is one of the following forms.

- First case: The walk $W^{\prime}$ is of the form

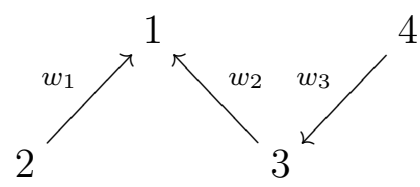

In this case $W$ has a subwalk of one of the following forms:

(i)

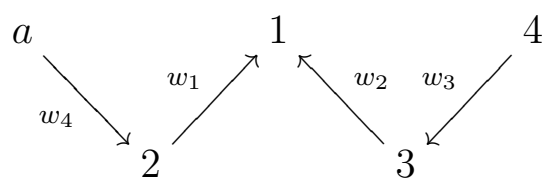

In this case the vertex $a$ can be either 1 or 2 or 5 . 
(ii)

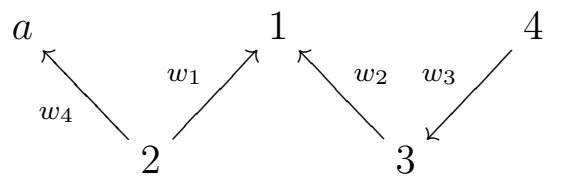

In this case the vertex $a$ can be either 2 or 3 or 4 or 5 .

(iii)

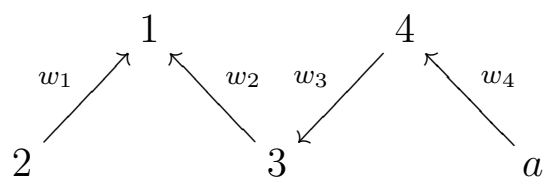

In this case the vertex $a$ can be either 1 or 3 or 4 or 5 . $(i v)$

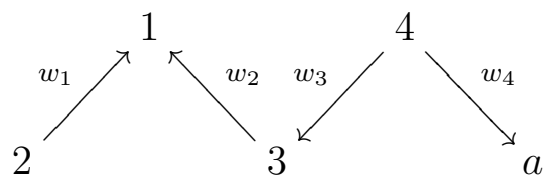

In this case the vertex $a$ can be either 2 or 4 or 5 .

- Second case: The walk $W^{\prime}$ is of the form

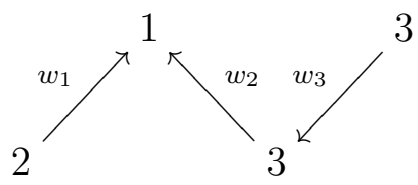

In this case $W$ has a subwalk of one of the following forms:

(i)

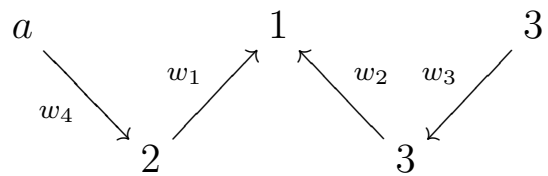

In this case the vertex $a$ can be either 1 or 2 or 4 .

(ii)

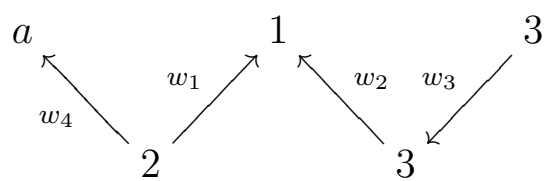

In this case the vertex $a$ can be either 2 or 4 .

(iii)

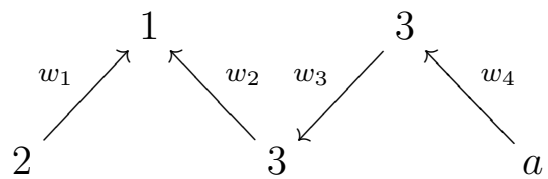

In this case the vertex $a$ can be either 1 or 4 .

- Third case: The walk $W^{\prime}$ is of the form 


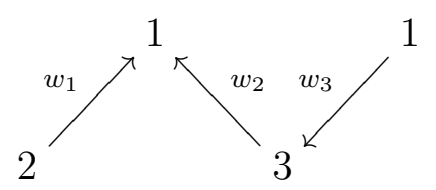

In this case $W$ has a subwalk of one of the following forms:

(i)

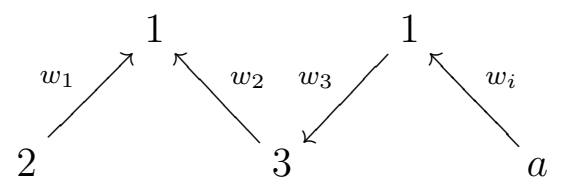

for $i=1,2$. If $i=1$, then $a=2$ and if $i=2$, then $a=3$.

(ii)

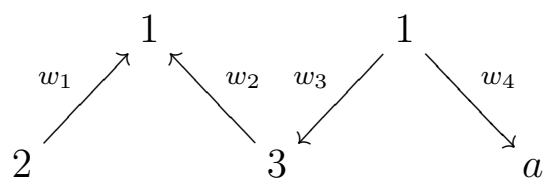
(iii)

In this case the vertex $a$ can be either 2 or 4 .

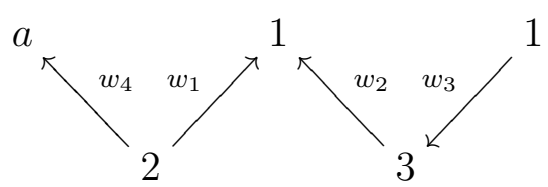

In this case the vertex $a$ can be either 2 or 3 or 4 . (iv)

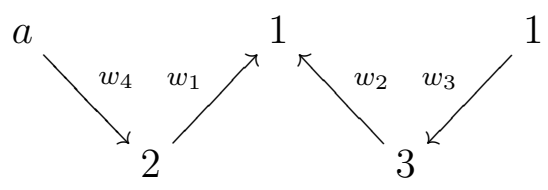

In this case the vertex $a$ can be either 2 or 4 .

- Forth case: The walk $W^{\prime}$ is of the form

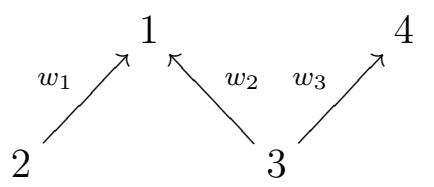

In this case $W$ has a subwalk of one of the following forms:

(i)

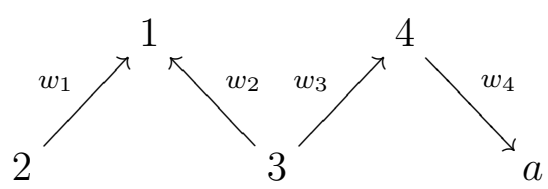

In this case the vertex $a$ can be either 2 or 3 or 4 or 5 . 
(ii)

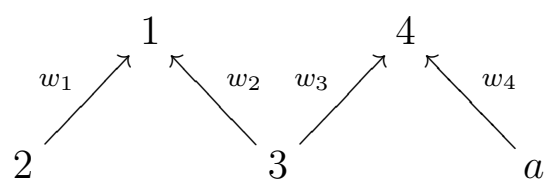

In this case the vertex $a$ can be either 1 or 4 or 5 .

(iii)

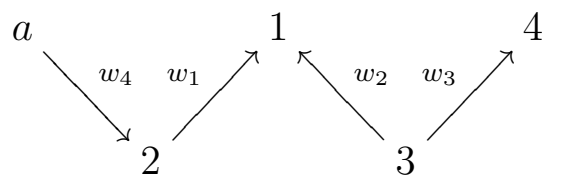

In this case the vertex $a$ can be either 1 or 2 or 5 .

(iv)

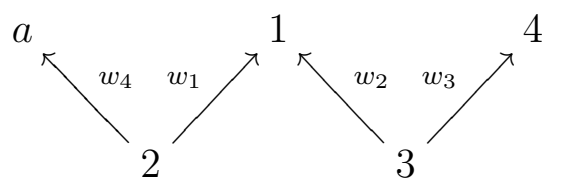

In this case the vertex $a$ can be either 2 or 3 or 5 .

- Fifth case: The walk $W^{\prime}$ is of the form

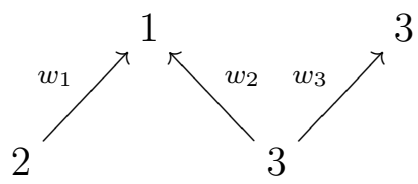

In this case $W$ has a subwalk of one of the following forms:

(i)

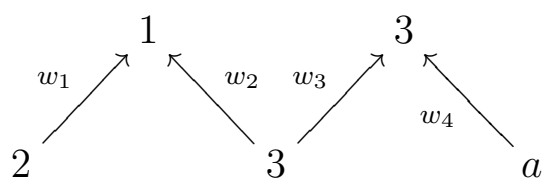

In this case the vertex $a$ can be either 1 or 4 .

(ii)

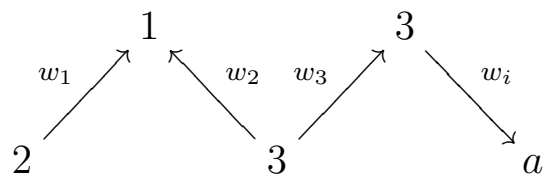

for $i=2,3$. If $i=2$, then $a=1$ and if $i=3$, then $a=3$.

(iii)

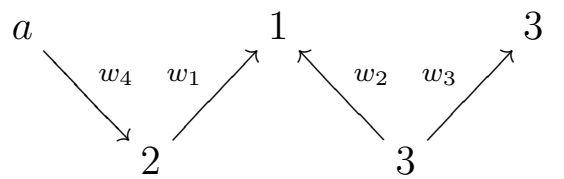

In this case the vertex $a$ can be either 1 or 2 or 4 . 
$(i v)$

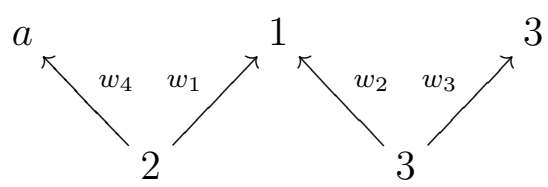

In this case the vertex $a$ can be either 2 or 4 .

- Sixth case: The walk $W^{\prime}$ is of the form

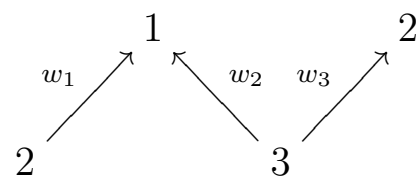

In this case $W$ has a subwalk of one of the following forms:

(i)

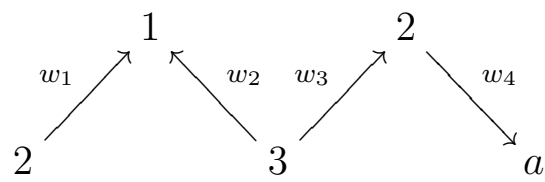

In this case the vertex $a$ can be either 3 or 4 .

(ii)

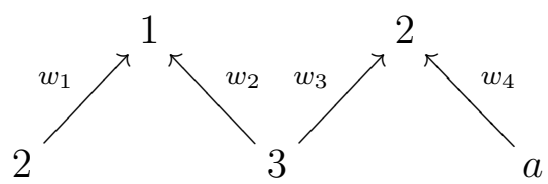

In this case the vertex $a$ can be either 1 or 4 .

- Seventh case: The walk $W^{\prime}$ is of the form

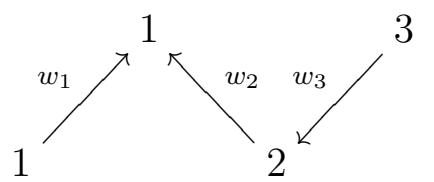

In this case $W$ has a subwalk of one of the following forms:

(i)

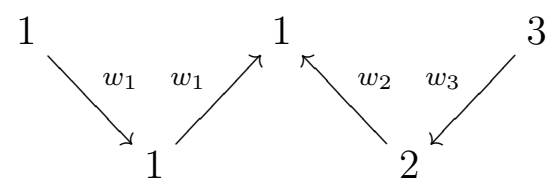

(ii)

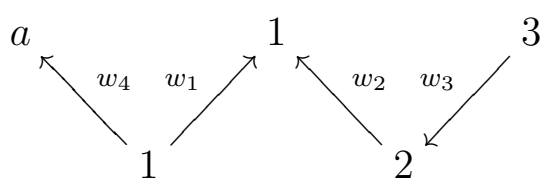

In this case the vertex $a$ can be either 2 or 3 or 4 . 
(iii)

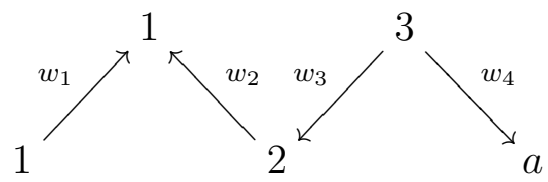

In this case the vertex $a$ can be either 3 or 4 .

$(i v)$

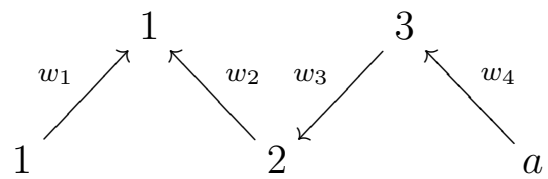

In this case the vertex $a$ can be either 2 or 3 or 4 .

- Eighth case: The walk $W^{\prime}$ is of the form

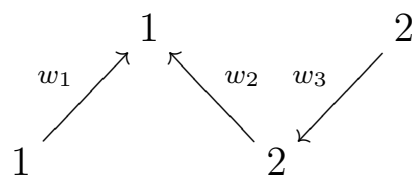

In this case $W$ has a subwalk of one of the following forms:

(i)

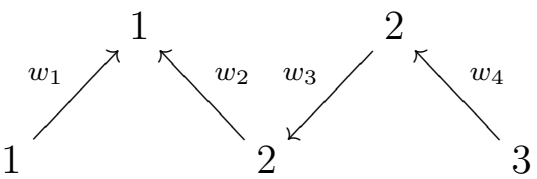

(ii)

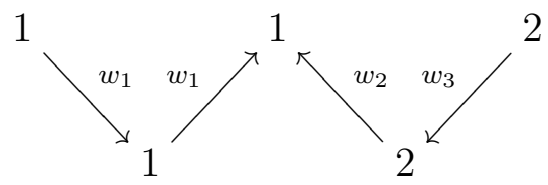

(iii)

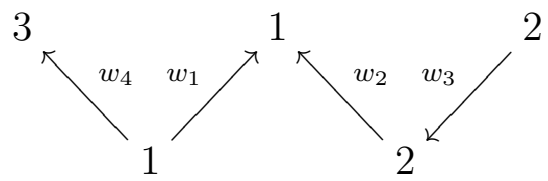

- Ninth case: The walk $W^{\prime}$ is of the form

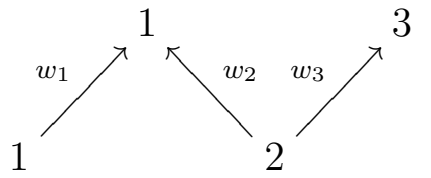

In this case $W$ has a subwalk of one of the following forms:

(i)

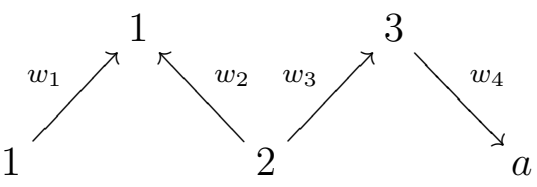


In this case the vertex $a$ can be either 2 or 3 or 4 .

(ii)

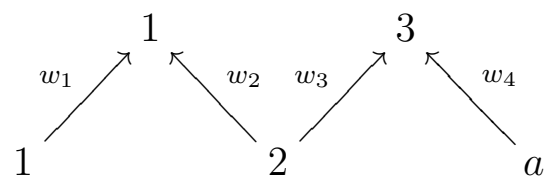

In this case the vertex $a$ can be either 3 or 4 .

(iii)

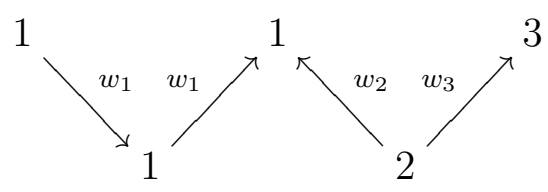

$(i v)$

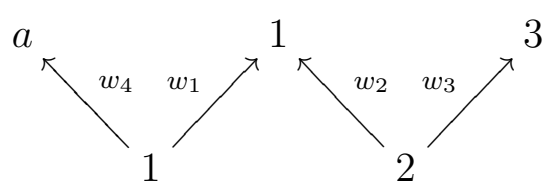

In this case the vertex $a$ can be either 2 or 4 .

- Tenth case: The walk $W^{\prime}$ is of the form

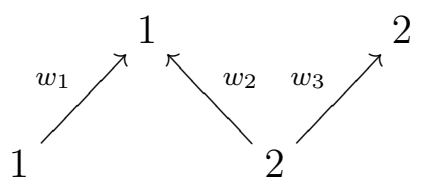

In this case $W$ has a subwalk of one of the following forms:

(i)

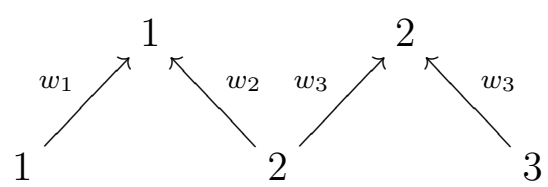

(ii)

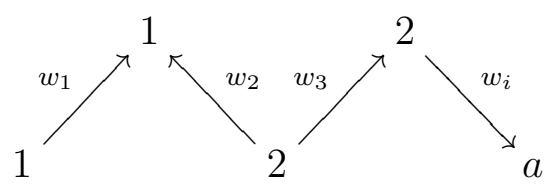

If $i=2$, then $a=1$ and if $i=3$, then $a=2$. (iii)

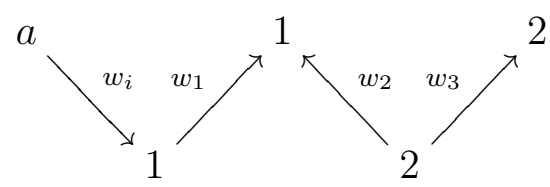

If $i=2$, then $a=2$ and if $i=1$, then $a=1$. 
$(i v)$

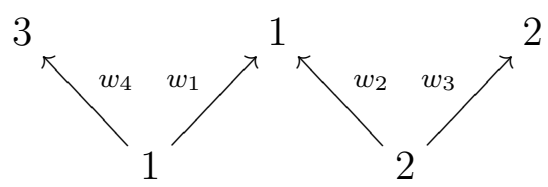

- Eleventh case: The walk $W^{\prime}$ is of the form

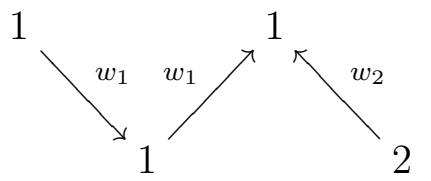

In this case $W$ has a subwalk of one of the following forms:

(i)

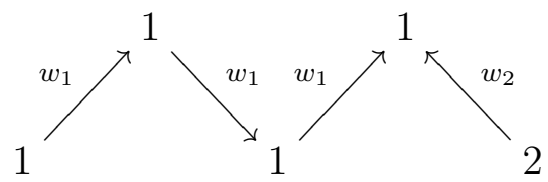

(ii)

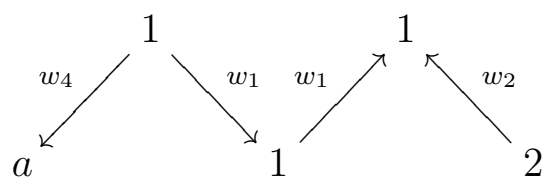

In this case the vertex $a$ can be either 2 or 3 .

(iii)

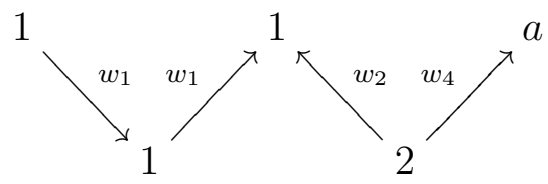

In this case the vertex $a$ can be either 2 or 3 .

(iv)

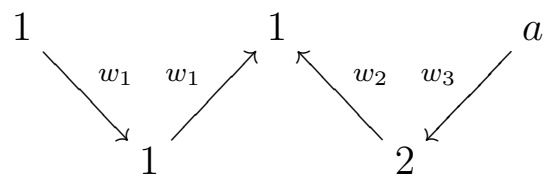

In this case the vertex $a$ can be either 2 or 3 .

- Twelfth case: The walk $W^{\prime}$ is of the form

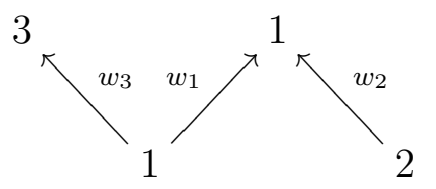

In this case $W$ has a subwalk of one of the following forms: 
(i)

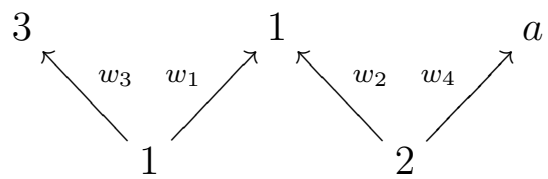

In this case the vertex $a$ can be either 2 or 4 .

(ii)

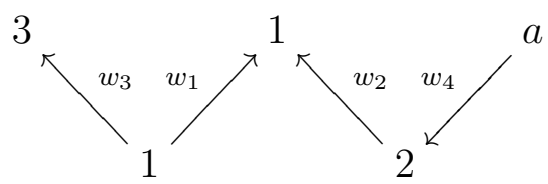

In this case the vertex $a$ can be either 2 or 3 or 4 .

(iii)

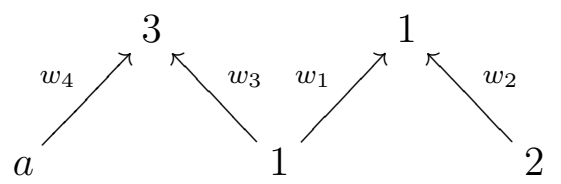

In this case the vertex $a$ can be either 3 or 4 .

$(i v)$

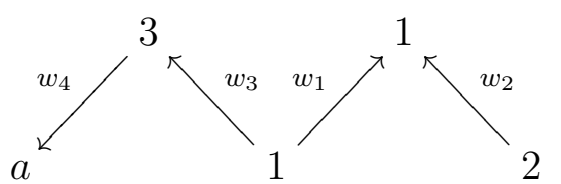

In this case the vertex $a$ can be either 2 or 3 or 4 .

- Thirteenth case: The walk $W^{\prime}$ is of the form

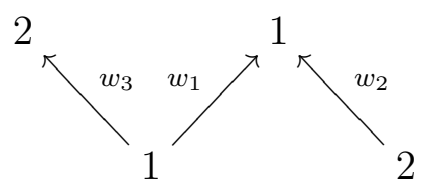

In this case $W$ has a subwalk of one of the following forms:

(i)

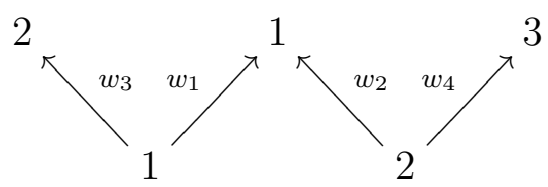

(ii)

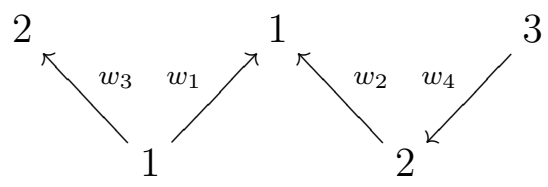

(iii)

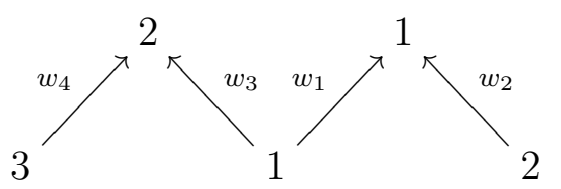


$(i v)$

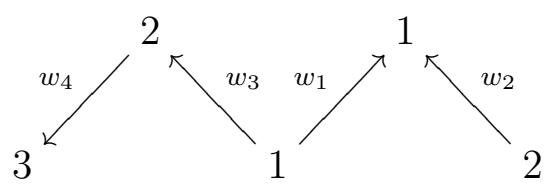

In all the above cases, there exists an indecomposable 5 -factor serial right $\Lambda$-module which gives a contradiction.

Assume on the contrary that the condition $(i i)$ does not hold. Then there exists a walk $W$ of length greater than or equal to 5 which has a subwalk $W^{\prime}$ of length 4 that $W^{\prime}$ has a subwalk of the form $w_{1}^{-1} w_{2}^{+1}$. By the condition $(i), W$ has not a subwalk $W^{\prime \prime}$ of length greater than or equal to 4 that $W^{\prime \prime}$ has a subwalk of the form $w_{1}^{+1} w_{2}^{-1}$. Since $\Lambda$ is an algebra of finite type, the walk $W^{\prime}$ is one of the following forms.

- First case: The walk $W^{\prime}$ is of the form

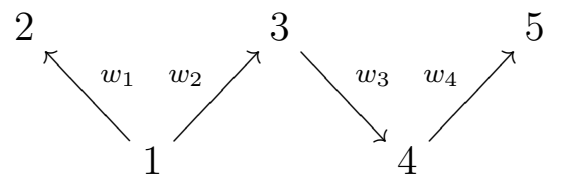

In this case $W$ has a subwalk of one of the following forms:

$(i)$

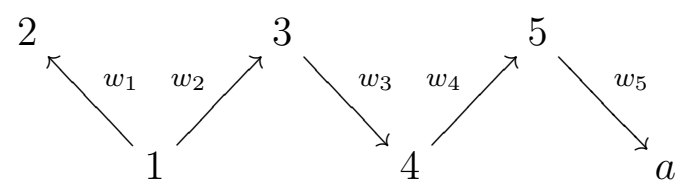

In this case the vertex $a$ can be either 6 or 1 .

(ii)

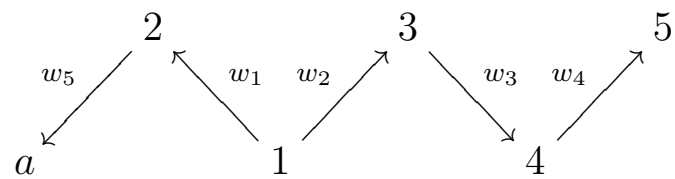

In this case the vertex $a$ can be either 6 or 1 .

- Second case: The walk $W^{\prime}$ is of the form

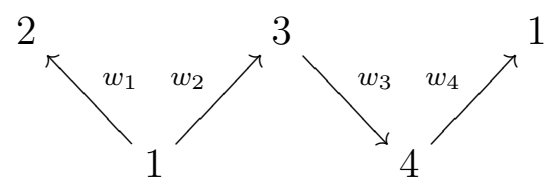

In this case $W$ has a subwalk of one of the following forms:

(i)

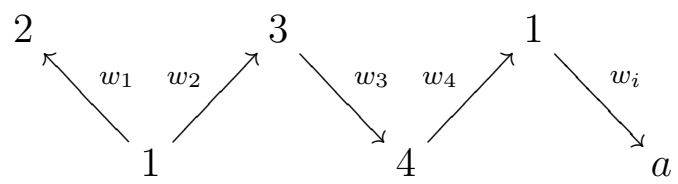

for $i=1,2$. If $i=1$, then $a=2$ and if $i=2$, then $a=3$. 
(ii)

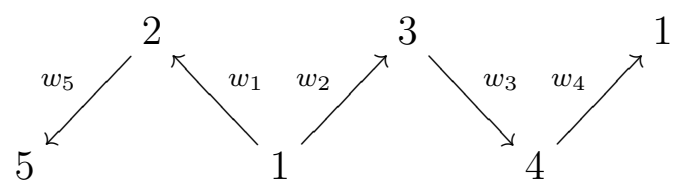

- Third case: The walk $W^{\prime}$ is of the form

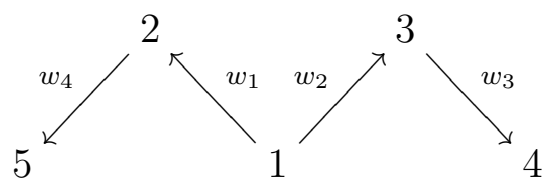

In this case $W$ has a subwalk of the following form:

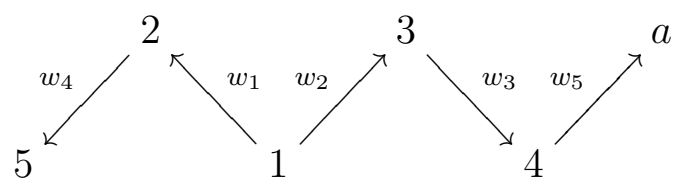

In this case the vertex $a$ can be either 6 or 1 .

- Fourth case: The walk $W^{\prime}$ is of the form

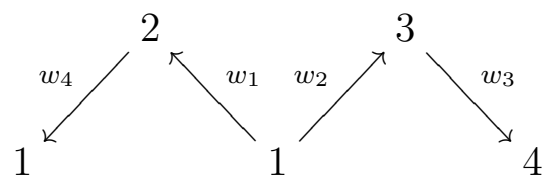

In this case $W$ has a subwalk of one of the following forms:

(i)

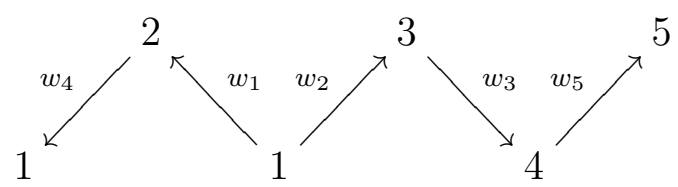

(ii)

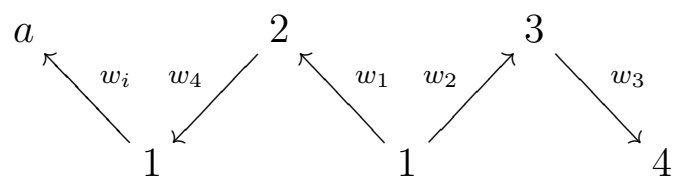

If $i=1$, then $a=2$ and if $i=2$, then $a=3$.

- Fifth case: The walk $W^{\prime}$ is of the form

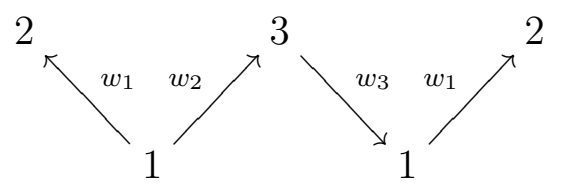

In this case $W$ has a subwalk of one of the following forms: 
(i)

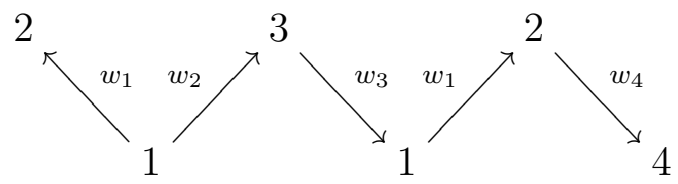

(ii)

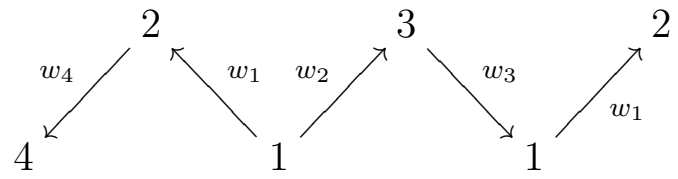

- Sixth case: The walk $W^{\prime}$ is of the form

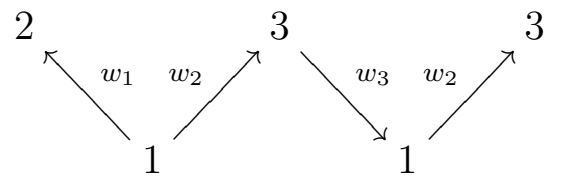

In this case $W$ has a subwalk of one of the following forms:

(i)

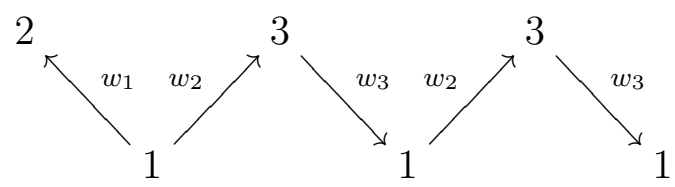

(ii)

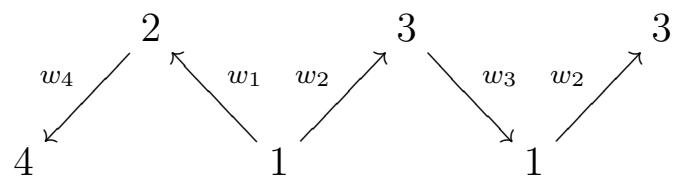

- Seventh case: The walk $W^{\prime}$ is of the form

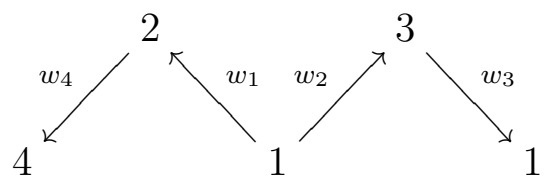

In this case $W$ has a subwalk of one of the following forms:

(i)

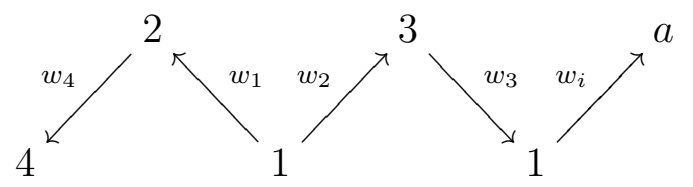

If $i=1$, then $a=2$ and if $i=2$, then $a=3$.

(ii)

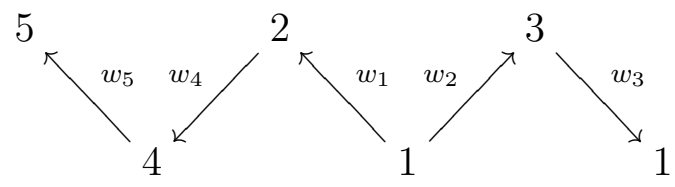


- Eighth case: The walk $W^{\prime}$ is of the form

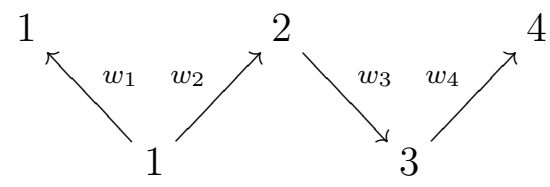

In this case $W$ has a subwalk of one of the following forms:

(i)

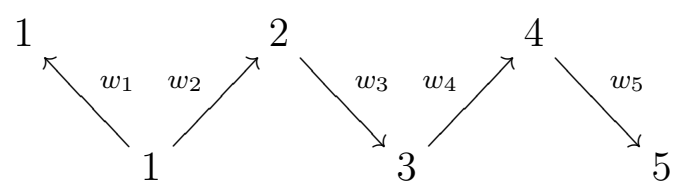

(ii)

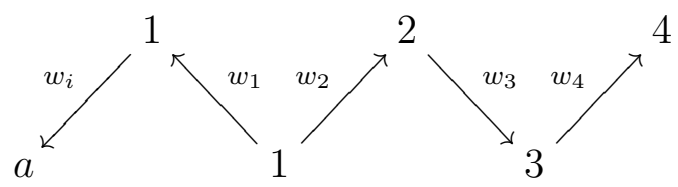

If $i=1$, then $a=1$ and if $i=2$, then $a=2$.

- Ninth case: The walk $W^{\prime}$ is of the form

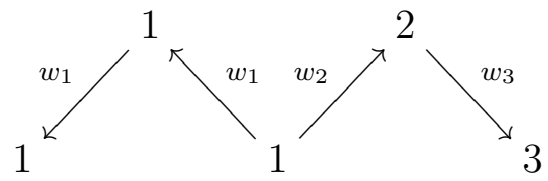

In this case $W$ has a subwalk of one of the following forms:

(i)

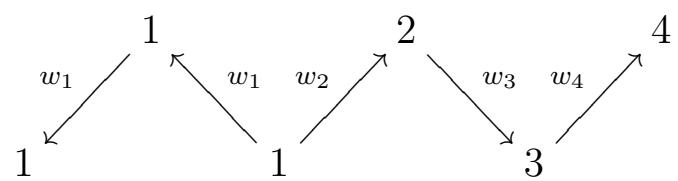

(ii)

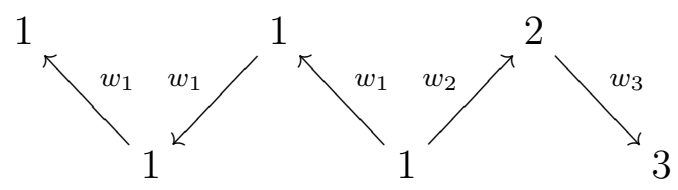

- Tenth case: The walk $W^{\prime}$ is of the form

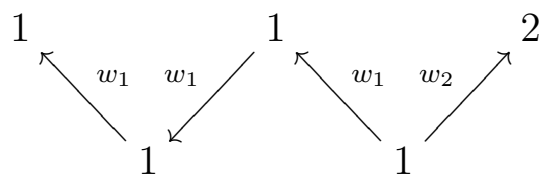

In this case $W$ has a subwalk of one of the following forms: 
(i)

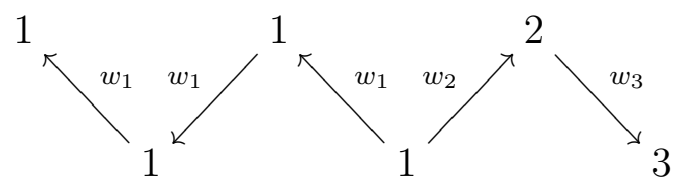

(ii)

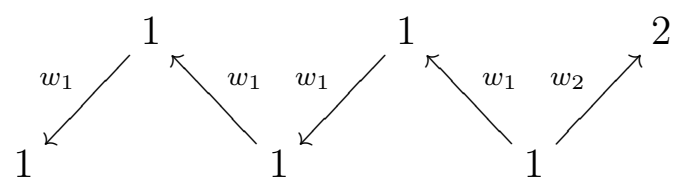

- Eleventh case: The walk $W^{\prime}$ is of the form

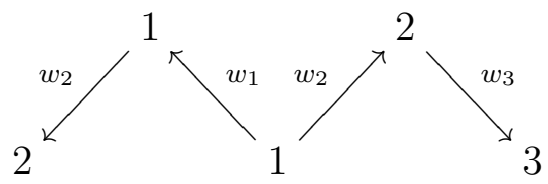

In this case $W$ has a subwalk of one of the following forms:

(i)

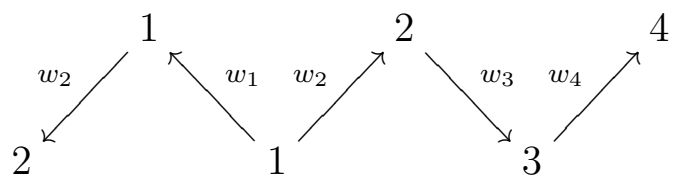

(ii)

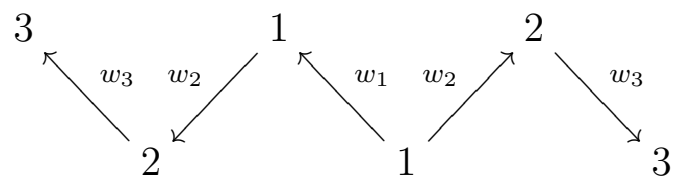

- Twelfth case: The walk $W^{\prime}$ is of the form

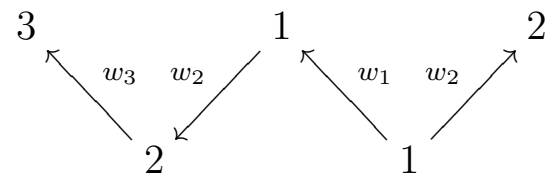

In this case $W$ has a subwalk of one of the following forms:

(i)

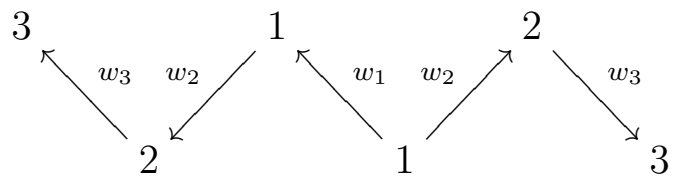

(ii)

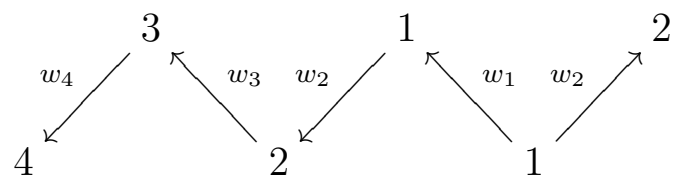


In all the above cases there exists an indecomposable 5 -factor serial right $\Lambda$-module which gives a contradiction.

Now assume that the condition ( $i i i)$ does not hold. Then there exist paths $p=p_{1} \ldots p_{t}$ and $q=q_{1} \ldots q_{s}$ with the same target and the same source that $p_{i}$ and $q_{j}$ are arrows, $s \geq 2$, $t \geq 2, p-q \in I$ and $s+t>5$.

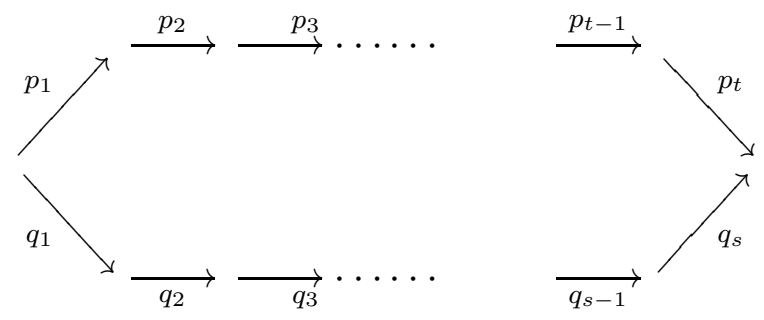

We have two cases. In the first case, we have $t \geq 2$ and $s \geq 4$. Then there exists a string $w=q_{s-2}^{+1} q_{s-1}^{+1} q_{s}^{+1} p_{t}^{-1}$ of $\frac{K Q}{I}$, that $M(w)$ is a 5 -factor serial right $\Lambda$-module which gives a contradiction. In the second case, we have $t \geq 3$ and $s \geq 3$. Then there exists a string $w=p_{t-1}^{+1} p_{t}^{+1} q_{s}^{-1} q_{s-1}^{-1}$ of $\frac{K Q}{I}$, that $M(w)$ is a 5 -factor serial right $\Lambda$-module which gives a contradiction. Now assume that the condition $(i v)$ does not hold. By [6, Theorem 5.13] and [5, Theorem 3.3] in this case $\Lambda$ is a $t$-Nakayama algebra for some $t \leq 3$, which gives a contradiction.

Conversely, assume that $\Lambda$ is a special biserial algebra of finite type that $(Q, I)$ satisfies the conditions $(i)-(i v)$. By [4] and [10], every indecomposable right $\Lambda$-module is either a string $M(w), w \in \widetilde{\mathcal{S}}(\Lambda)$ or a band module $M(v, m, \varphi), v \in \widetilde{\mathcal{B}}(\Lambda), m \geq 1$ and $\varphi \in$ $\operatorname{Aut}\left(K^{m}\right)$ or non-uniserial projective-injective. Since $\Lambda$ is representation-finite, $\mathcal{B}(\Lambda)=$ $\varnothing$. For any $w \in \widetilde{\mathcal{S}}(\Lambda), w$ is either $w_{1}^{+1} \ldots w_{n}^{+1}$ or $w_{1}^{-1} w_{2}^{+1}$ or $w_{1}^{+1} w_{2}^{-1}$ or $w_{1}^{-1} w_{2}^{-1} w_{3}^{+1}$ or $w_{1}^{-1} w_{2}^{-1} w_{3}^{+1} w_{4}^{+1}$ or $w_{1}^{-1} w_{2}^{+1} w_{3}^{+1} w_{4}^{+1}$ or $w_{1}^{+1} w_{2}^{+1} w_{3}^{-1}$ or $w_{1}^{-1} w_{2}^{+1} w_{3}^{-1}$. If $w=w_{1}^{+1} \ldots w_{n}^{+1}$, then $M(w)$ is uniserial. If $w=w_{1}^{-1} w_{2}^{+1}$, then $M(w)$ is 2-factor serial. If either $w=$ $w_{1}^{+1} w_{2}^{-1}$ or $w=w_{1}^{-1} w_{2}^{-1} w_{3}^{+1}$, then $M(w)$ is 3-factor serial. If either $w_{1}^{-1} w_{2}^{-1} w_{3}^{+1} w_{4}^{+1}$ or $w_{1}^{-1} w_{2}^{+1} w_{3}^{+1} w_{4}^{+1}$ or $w_{1}^{+1} w_{2}^{+1} w_{3}^{-1}$ or $w_{1}^{-1} w_{2}^{+1} w_{3}^{-1}$, then $M(w)$ is a 4 -factor serial right $\Lambda$ module. By the condition (iv), there exists at least one string module $M(w)$, where $w$ is either $w_{1}^{-1} w_{2}^{-1} w_{3}^{+1} w_{4}^{+1}$ or $w_{1}^{-1} w_{2}^{+1} w_{3}^{+1} w_{4}^{+1}$ or $w_{1}^{+1} w_{2}^{+1} w_{3}^{-1}$ or $w_{1}^{-1} w_{2}^{+1} w_{3}^{-1}$. Therefore $\Lambda$ is a right 4 -Nakayama and the result follows.

\section{Remark 5.3.}

(1) Let $\Lambda=K Q / I$ be a right 4-Nakayama finite dimensional $K$-algebra that the condition $(i v)(c)$ of the theorem 5.2 holds. Then there exists a non-uniserial projective-injective right $\Lambda$-module $M$, that $M$ is either 3 -factor serial or 4 -factor serial.

(2) Let $\Lambda=\frac{K Q}{I}$ be a basic, connected and finite dimensional $K$-algebra. By [5, Proposition 4.2] $\Lambda$ is right 4-Nakayama self-injective if and only if $Q$ is the following quiver with $s \geq 1$ 
and $m, n \geq 2$,

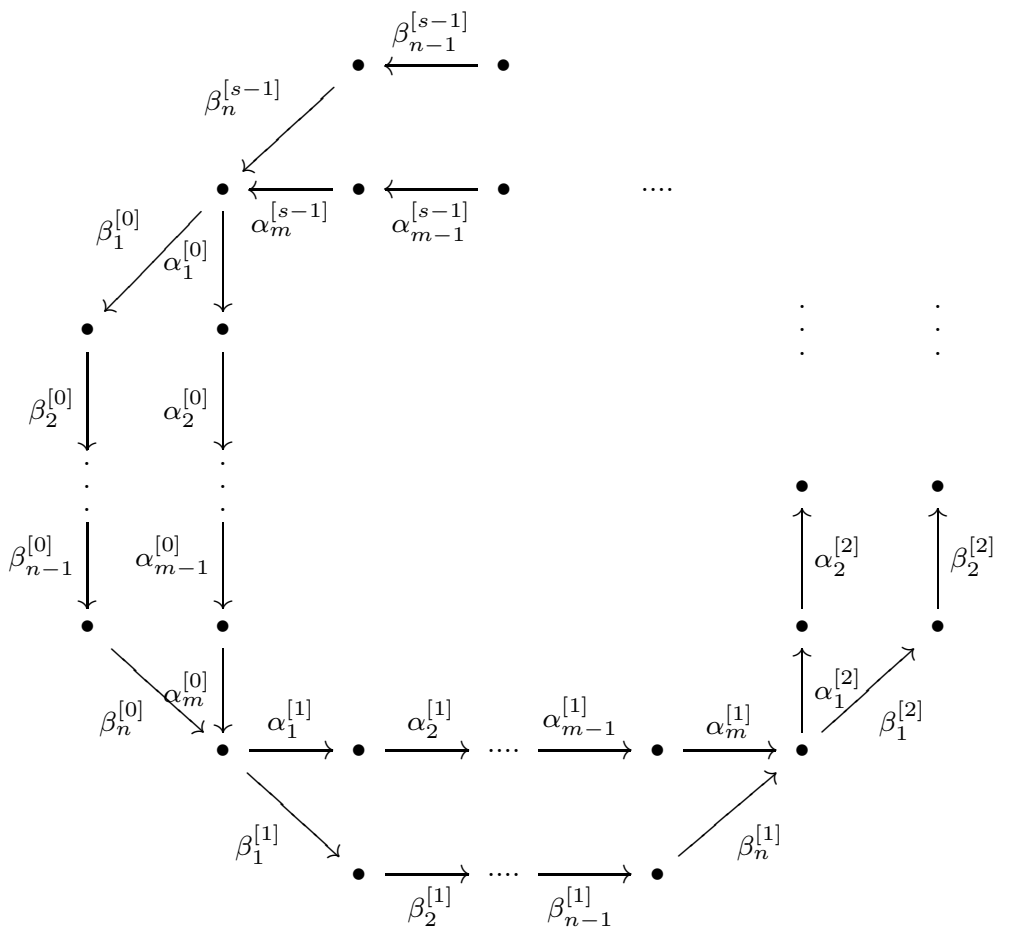

and $I$ is the ideal generated by the following relations:

(i) $\alpha_{1}^{[i]} \cdots \alpha_{m}^{[i]}=\beta_{1}^{[i]} \cdots \beta_{n}^{[i]}$ for all $i \in\{0, \cdots, s-1\}$;

(ii) $\beta_{n}^{[i]} \alpha_{1}^{[i+1]}=0, \alpha_{m}^{[i]} \beta_{1}^{[i+1]}=0$ for all $i \in\{0, \cdots, s-2\}, \beta_{n}^{[s-1]} \alpha_{1}^{[0]}=0$ and $\alpha_{m}^{[s-1]} \beta_{1}^{[0]}=$ 0

(iii) (a) Paths of the form $\alpha_{i}^{[j]} \ldots \alpha_{h}^{[f]}$ of length $m+1$ are equal to 0 .

(b) Paths of the form $\beta_{i}^{[j]} \ldots \beta_{h}^{[f]}$ of length $n+1$ are equal to 0.

that $m+n=5$.

\section{ACKNOWLEDGEMENTS}

The research of the first author was in part supported by a grant from IPM (No. 96170419).

\section{REFERENCES}

[1] H. Asashiba, On algebras of second local type II, Osaka J. Math. 21 (1984), 343-364.

[2] H. Asashiba, On algebras of second local type III, Osaka J. Math. 24 (1987), 107-122.

[3] M. Auslander, I. Reiten, S. O. Smal $\varnothing$, Representation theory of Artin algebras, Cambridge studies in advanced mathematics 36, Cambridge University Press, Cambridge, 1995.

[4] M. C. R. Butler, C. M. Ringel, Auslander-Reiten sequences with few middle terms, with applications to string algebras, Comm. Algebra 15 (1987), 145-179.

[5] A. NASR-Isfahani, M. SheKari, Representations of right 3-Nakayama algebras, arXiv:1805.04412,

[6] A. NASR-ISFAhAnI, M. SheKARI, Right $n$-Nakayama algebras and their representations, arXiv:1710.01176.

[7] A. Skowronski, K. Yamagata, Frobenius algebras I: Basic representation theory, EMS Textbooks in Mathematics, European Mathematical Society (EMS), Zurich, 2011. 
[8] H. TAChikawa, On algebras of which every indecomposable representation has an irreducible one as the top or the bottom Loewy constituent, Math. Z. 75 (1961), 215-227.

[9] H. TACHIKAWA, On rings for which every indecomposable right module has a unique maximal submodule, Math. Z. 71 (1959), 200-222.

[10] B. Wald, J. Waschbusch, Tame biserial algebras, J. Algebra 15 (1985), no. 2, 480-500.

[11] A. Zimmermann, Representation theory, A homological algebra point of view, Algebra and Applications, vol. 19, Springer, Cham, 2014.

Department of Mathematics, University of Isfahan, P.O. Box: 81746-73441, Isfahan, Iran, and School of Mathematics, Institute for Research in Fundamental Sciences (IPM), P.O. Box: 19395-5746, Tehran, Iran

E-mail address: nasr_a@sci.ui.ac.ir / nasr@ipm.ir

Department of Mathematics, University of Isfahan, P.O. Box: 81746-73441, Isfahan, IRAN

E-mail address: mshekari@sci.ui.ac.ir 